2001s-54

\title{
Testing for Structural Change in the Presence of Auxiliary Models
}

Eric Ghysels, Alain Guay

Série Scientifique

Scientific Series

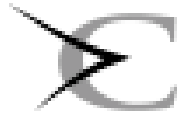

CIRANO

Ceatre intereaiversitaire de recherche
en ansalyne des organisaticas

Montréal

Septembre 2001 


\section{CIRANO}

Le CIRANO est un organisme sans but lucratif constitué en vertu de la Loi des compagnies du Québec. Le financement de son infrastructure et de ses activités de recherche provient des cotisations de ses organisationsmembres, d'une subvention d'infrastructure du ministère de la Recherche, de la Science et de la Technologie, de même que des subventions et mandats obtenus par ses équipes de recherche.

CIRANO is a private non-profit organization incorporated under the Québec Companies Act. Its infrastructure and research activities are funded through fees paid by member organizations, an infrastructure grant from the Ministère de la Recherche, de la Science et de la Technologie, and grants and research mandates obtained by its research teams.

\section{Les organisations-partenaires / The Partner Organizations}

-École des Hautes Études Commerciales

-École Polytechnique

-Université Concordia

-Université de Montréal

-Université du Québec à Montréal

-Université Laval

-Université McGill

-Ministère des Finances du Québec

-MRST

-Alcan inc.

-AXA Canada

-Banque du Canada

-Banque Laurentienne du Canada

-Banque Nationale du Canada

-Banque Royale du Canada

-Bell Québec

-Bombardier

-Bourse de Montréal

-Développement des ressources humaines Canada (DRHC)

-Fédération des caisses Desjardins du Québec

-Hydro-Québec

-Industrie Canada

-Pratt \& Whitney Canada Inc.

-Raymond Chabot Grant Thornton

-Ville de Montréal

(C) 2001 Eric Ghysels et Alain Guay. Tous droits réservés. All rights reserved.

Reproduction partielle permise avec citation du document source, incluant la notice $\odot$.

Short sections may be quoted without explicit permission, if full credit, including $\odot$ notice, is given to the source.

Ce document est publié dans l'intention de rendre accessibles les résultats préliminaires

de la recherche effectuée au CIRANO, afin de susciter des échanges et des suggestions.

Les idées et les opinions émises sont sous l'unique responsabilité des auteurs, et ne représentent pas nécessairement les positions du CIRANO ou de ses partenaires.

This paper presents preliminary research carried out at CIRANO and aims at encouraging discussion and comment. The observations and viewpoints expressed are the sole responsibility of the authors. They do not necessarily represent positions of CIRANO or its partners. 


\title{
Testing for Structural Change in the Presence of Auxiliary Models*
}

\author{
Eric Ghysels ${ }^{\dagger}$, Alain Guay
}

\section{Résumé / Abstract}

Plusieurs méthodes d'estimation nécessitent un modèle instrumental et un modèle d'intérêt. On retrouve parmi ces méthodes la méthode des moments efficace de Gallant et Tauchen (1996) et l'Inférence Indirecte proposée par Gouriéroux, Monfort et Renault (1993). La présence de ces deux modèles procure de nouvelles occasions d'inférence. Dans cet article, on présente et dérive la loi asymptotique de différents tests de changement structurel. Certaines procédures sont des extensions de tests standards tandis que d'autres sont spécifiquement adaptées à la présence des deux modèles.

Several estimation procedures such as the Efficient Method of Moments (EMM) of Gallant and Tauchen (1996) and Indirect Inference procedure of Gouriéroux, Monfort and Renault (1993) involve two models, an auxiliary one and a model of interest. The role played by both models poses challenges and provides new opportunities for hypothesis testing beyond the usual Wald, LM and LR-type tests. In this paper we present the asymptotic distribution theory for various classes of tests for structural change. Some procedures are extensions of standard tests while others are specific to the dual model setup and exploit its unique features.

Mots Clés : Méthodes simulées d'estimations, test de stabilité structurelle, tests optimaux

Keywords: Simulated method of estimation, structural stability testing, optimal tests

JEL : C1, C12, C22

\footnotetext{
* Corresponding Author: Alain Guay, CREFE, UQAM, CP 8888, Succursale Centre-ville, Montréal, Qc, Canada H3C 3P8 email: guay.alain@uqam.ca

We wouls like to acknowledge the financial support of the Natural Sciences and Engineering Research Council of Canada through a grant to NCM2 [Network for Computing and Mathematical Modeling]. We would also like to thank Alastair Hall and Éric Renault for comments on an earlier draft of the paper.

${ }^{\dagger}$ University of North Carolina and CIRANO

‡ Université du Québec à Montréal and CREFE
} 


\section{Introduction}

There is now a fully developed asymptotic distribution theory for various types of test statistics associated with Generalized Method of Moments (henceforth GMM) and Simulated Method of Moments (henceforth SMM) estimators. The seminal paper by Hansen (1982) on GMM proposed a widely used test for overidentifying restrictions, while Gallant (1987) and Newey and West (1988) presented generic Wald, LM and LR-type tests. Andrews and Ploberger (1994) deal with optimal tests when a nuisance parameter is present only under the alternative. One of the most prominent applications of such test statistics involves the hypothesis of structural change with unknown breakpoint. ${ }^{1}$ McFadden (1989), Pollard and Pakes (1989) and Duffie and Singleton (1993) extended the GMM framework to estimation methods involving simulated moments. A comprehensive treatment of Wald, LM and LRtype tests for SMM can be found in Gouriéroux, Monfort and Renault (1993).

In recent years a number of estimation procedures have been proposed which involve a dual model setup. Examples include Asymptotic Least Squares (henceforth ALS) of Gouriéroux, Monfort and Trognon (1985), the indirect inference method of Gouriéroux et al. (1993) (henceforth I.I.) and the Efficient Method of Moments (called EMM) procedure of Gallant and Tauchen (1996). Estimation procedures involving auxiliary models are more commonly used, particularly in situations where likelihood-based estimation or method of moments are infeasible. Many empirical examples can be found in macroeconomic and financial econometrics literature. These procedures are driven by the fundamental distinction between an auxiliary model, parameterized by a vector $\theta$, and a model of interest, which is parameterized by $\rho$. The distinct role played by both models can be viewed as adding complications to the formulation of traditional tests and can also be viewed as the basis for formulating new classes of tests. The purpose of our paper is to examine both issues.

We present several classes of tests for structural change, some are extensions of tests proposed for GMM and SMM while others genuinely exploit features unique to the dual model setup. We proceed in two steps. First we ignore the simulation uncertainty and deal with tests for structural change in a GMM-type setup involving an auxiliary model. Such tests are based on the ALS principle. Next we add the simulation uncertainty and present a generic class of tests for structural change with unknown breakpoints for EMM and I.I.

\footnotetext{
${ }^{1}$ It should be noted that various tests for the structural change hypothesis were developed for the GMM estimator; see for instance Andrews and Fair (1988), Dufour, Ghysels and Hall (1994), Ghysels, Guay and Hall (1997), Ghysels and Hall (1990), Guay (1996), Hall and Sen (1999), Hoffman and Pagan (1989), Sowell (1996a), among others.
} 
estimators.

Among the tests for structural change specifically tailored for EMM and I.I. figures a class of tests based on a principle of simulated scores which is specific to the combination of an auxiliary model and simulation based estimation. The simulated score tests we propose use simulated series from a restricted null model of interest. Using the reprojection arguments of Gallant and Tauchen (1998) we can fit a sieve seminonparametric SNP density to the simulated data. Under the null the simulated data should yield a reprojection score generator which is a martingale difference sequence when applied to the actual sample data. To test the martingale difference hypothesis we consider tests proposed by Bierens and Ploberger (1997) and de Jong (1996). We extend their tests to the simulation-based context of EMM and I.I. Hence it is a test principle tailored exclusively for EMM and I.I. applied here in the context of structural change.

Our analysis also relates to a EMM diagnostic test proposed by Liu and Zhang (1998). Their test, while meant to be a simulated score test, is closely related to one of the structural change tests we propose. We generalize and extend the test Liu and Zhang (1998) suggested. Recent work by van der Sluis (1998) also proposes structural change tests for EMM. We show that the asymptotic derivations in van der Sluis are invalid for the proposed statistics and compare our tests with the Hansen J-type and Hall-Sen type tests discussed in van der Sluis (1998).

The paper is organized as follows: In section 2 we discuss tests for structural change with unknown breakpoint. Section 3 deals with simulated score tests. Section 4 covers non-nested hypothesis testing while section 5 concludes.

\section{Models and Parameter Estimators}

In this section we describe the data generating processes as well as the various classes of estimators we will consider. A first subsection is devoted to the description of the data generating processes. The second subsection covers the parameter estimators.

\subsection{The Data Generating Processes}

The data generating process is described by a parametric nonlinear simultaneous equations model, namely:

$$
r\left(y_{t}, y_{t-1}, x_{t}, u_{t}, \rho\right)=0
$$




$$
q\left(u_{t}, u_{t-1}, \varepsilon_{t}, \rho\right)=0
$$

where $\rho \in \Re \subset R^{p},\left\{y_{t}\right\}$ corresponds to the vector of dependent variables whereas $\left\{x_{t}\right\}$ is the vector of exogenous variables. Both vector processes are stationary and observable, in addition $\left\{x_{t}\right\}$ is a homogeneous Markov process independent of $\left\{\varepsilon_{t}\right\}$ and $\left\{u_{t}\right\}$. The latter two are latent processes with $\varepsilon_{t}$ white noise with known distribution $G_{0} \cdot{ }^{2}$ The fact that only one lag is considered in (2.1) and (2.2) is not essential and can easily be relaxed. ${ }^{3}$ It will also be convenient to define the vector $Z_{t-1} \equiv\left(y_{t-1}, x_{t}\right)$. Equations $(2.1)$ and (2.2) correspond to the data generating processes considered by Gouriéroux et al. (1993), Gallant and Tauchen (1996) and Broze et al. (1998). Since we will be dealing with simulation-based estimators we assume that samples of simulated $\left\{y_{t}^{s}(\rho)\right\}_{t=1}^{T}$ can be generated uniquely through (2.1) and (2.2), given $\rho$ and conditional on initial values $u_{0}$ and $y_{0}$ as well as the observed path of exogenous variables $\left\{x_{t}\right\}_{t=1}^{T}$.

The indirect inference method of Gouriéroux et al. (1993) and the efficient method of moments of Gallant and Tauchen (1996) are estimation procedures designed for situations where the log-likelihood function of the structural model:

$$
\zeta_{T}(\rho)=\sum_{t=1}^{T} \log \ell\left(y_{t} \mid Z_{t-1}, \rho\right)
$$

is computationally intractable. The likelihood-based method is therefore replaced by an instrumental criterion which involves a vector of parameters $\theta \in \Theta \subset R^{q}$, namely:

$$
Q_{T}(\theta)=\sum_{t=1}^{T} \psi_{t}\left(y_{t} \mid Z_{t-1}, \theta\right) .
$$

Minimizing (2.4) yields an M-estimator $\widehat{\theta}_{T}$ for $\theta$. The auxiliary model parameters $\theta$ and those of the structural model are related through:

$$
g\left(\theta^{*}(\rho), \rho^{0}\right)=0
$$

where $\rho^{0}$ is the true value and $\theta^{*}$ is the estimator which minimizes the limit $($ as $T \rightarrow \infty)$ of the M-estimation criterion (2.4). Equation (2.5) yields a so-called binding function $\theta^{*}=b\left(\rho^{0}\right)$. The I.I. and EMM procedures provide, in different ways, simulation-based approximations to the binding function. Moreover, the function in (2.5) must satisfy:

\footnotetext{
${ }^{2}$ The assumption of white noise can be relaxed, see Gouriéroux et al. (1993) for further discussion.

${ }^{3}$ In principle an infinite number of lags can be considered as discussed by Gallant and Tauchen (1996).
} 
Assumption 2.1 For the purpose of identification, $p \leq G \leq q$ in (2.5), where $\rho \in \Re \subset R^{p}$, $\theta \in \Theta \subset R^{q}, g \in R^{G}$ and $G_{\theta}=\partial g\left(\theta^{*}, \rho^{0}\right) / \partial \theta^{\prime}$ is of full column rank.

Finally, it will be useful to split the parameter vector $\rho$ into two subvectors $\rho=\left(\rho^{1}, \rho^{2}\right)$. There are at least two motivating reasons for this. First, following Andrews (1993) one can consider tests for partial structural change where only a subvector $\rho^{1}$ of the parameter vector of interest $\rho$ is tested for structural change. Second, following Broze et al. (1998) and Dridi and Renault (2001) one can also consider situations where only a subvector $\rho^{1}$ of $\rho$ is of direct interest while $\rho^{2}$ consists of nuisance parameters, such as parameters pertaining for instance to distributional assumptions. Such a situation, which Broze and al. (1998) and Dridi and Renault (2001) label semiparametric indirect inference, also suggests tests for structural change for subvectors corresponding to parameters of economic interest. Throughout the remainder of this paper we will discuss the implications of partial structural change and semiparametric indirect inference. To keep the notational complexity minimal, we avoid spliting the parameter vector $\rho$ in subvectors. All the results we present can easily be modified to take into account the special cases of testing the null hypothesis of structural change for subvectors.

\subsection{Parameter estimators}

The Asymptotic Least Squares estimator of Gouriéroux, Monfort and Trognon (1985) is a procedure for estimating $\rho$ through an auxiliary model parameterized by $\theta$. Its main advantage, which we exploit here for expository purpose, is that it does not involve simulation uncertainty. Sidestepping this source of uncertainty, at least at a first stage, allows us to focus first and foremost on the key issue of testing for structural change when an auxiliary model is present.

\subsubsection{The Asymptotic Least Squares estimator}

We will consider several ALS estimators. In particular, we define the estimator for the entire sample of the parameter vector of the auxiliary model as the following M-estimator:

$$
\hat{\theta}_{T}=\arg \min _{\theta \in \Theta} \frac{1}{T} \sum_{t=1}^{T} \psi_{t}\left(y_{t} \mid Z_{t-1} ; \theta\right)
$$

where $\theta \in \Theta \subset R^{q}$. Some tests for structural change involve parameter estimators over subsamples. We will call full sample estimators, like (2.6), as restricted estimators since the parameters are assumed identical across subsamples. To define an unrestricted estimator we 
consider explicitly two subsamples, the first is based on observations $t=1, \cdots,[T \pi]$ while the second subsample covers $t=[T \pi]+1, \cdots, T$ where $\pi \in \Pi \subset(0,1)$. The separation $[T \pi]$ represents a possible breakpoint and [.] denotes the greatest integer function. The unrestricted asymptotic least squares estimators for the first and the second subsamples are,

$$
\begin{gathered}
\hat{\theta}_{1 T}(\pi)=\arg \min _{\theta_{1} \in \Theta} \frac{1}{[T \pi]} \sum_{t=1}^{[T \pi]} \psi_{t}\left(y_{t} \mid Z_{t-1} ; \theta\right) \\
\hat{\theta}_{2 T}(\pi)=\arg \min _{\theta_{2} \in \Theta} \frac{1}{T-[T \pi]} \sum_{t=[T \pi]+1}^{T} \psi_{t}\left(y_{t} \mid Z_{t-1} ; \theta\right) .
\end{gathered}
$$

To simplify the notation, we define $\Psi_{1 T}(\theta, \pi)=\frac{1}{[T \pi]} \sum_{t=1}^{[T \pi]} \psi_{t}\left(y_{t} \mid Z_{t-1} ; \theta_{1}\right), \Psi_{2 T}(\theta, \pi)=$ $\frac{1}{T-[T \pi]} \sum_{t=[T \pi]+1}^{T} \psi_{t}\left(y_{t} \mid Z_{t-1} ; \theta\right)$ and the vector $\Psi_{T}(\theta, \pi)=\left(\Psi_{1 T}(\theta, \pi), \Psi_{2 T}(\theta, \pi)\right)$. The unrestricted least square estimators of the parameter vector $\rho$ for the first and the second subsamples are obtained by:

$$
\hat{\rho}_{i T}(\pi)=\arg \min _{\rho_{i} \in \Re} g\left(\hat{\theta}_{i T}(\pi), \rho\right)^{\prime} W_{i T}(\pi) g\left(\hat{\theta}_{i T}(\pi), \rho\right)
$$

where $W_{i T}$ are random nonnegative symmetric matrices and $g$ is defined in (2.5). The corresponding binding functions are $\theta_{1}^{*}(\pi)=b_{1}\left(\rho_{1}^{0}, \pi\right)$ and $\theta_{2}^{*}(\pi)=b_{2}\left(\rho_{2}^{0}, \pi\right)$ and $\theta_{i}^{*}$ is the estimator which minimizes the limit $($ as $T \rightarrow \infty$ ) for each of the subsamples $i=1,2$. The restricted asymptotic least squares estimator for $\rho$ is obtained via a function relating $\theta^{*}$ to the parameter of interest. This function is defined as $g\left(\theta^{*}(\rho), \rho^{0}\right)=0$ where $\hat{\theta}$ replaces $\theta^{*}$, i.e. the estimator which minimizes as $T \rightarrow \infty$ the limit of the M-criterion. Hence, the restricted (i.e. full sample) estimator is:

$$
\hat{\rho}_{T}=\arg \min _{\rho \in \Re} g\left(\hat{\theta}_{T}, \rho\right)^{\prime} W_{T} g\left(\hat{\theta}_{T}, \rho\right)
$$

where $W_{T}$ is a random nonnegative symmetric matrix.

\subsubsection{The Indirect Inference estimator}

The indirect inference method of Gouriéroux et al. (1993) also involves the binding function which relates the estimator for the auxiliary model to the estimator of the structural model $\theta^{*}=b\left(\rho^{0}\right)$. The binding function is unknown, however, and therefore is approximated by simulation. Assume one selects a value of $\rho$ and, using equations (2.1) and (2.2), one simulates the process $\left\{y_{t}^{s}(\rho)\right\}_{t=1}^{T}$. The estimator of the auxiliary model is then defined as:

$$
\left.\hat{\theta}_{T}^{s}(\rho)=\arg \min _{\theta \in \Theta} \frac{1}{T} \sum_{t=1}^{T} \psi_{t}\left(y_{t}^{s}(\rho) \mid Z_{t-1}^{s}(\rho), \theta\right)\right)
$$


where $Z_{0}^{s}$ are the initial values $\left(y_{-1}, x_{0}\right)$ for the $s$ simulated path. Note also that $Z_{t-1}^{s}(\rho) \equiv$ $\left(y_{t-1}^{s}(\rho), x_{t}\right)$. For $S$ simulated paths, we construct $\frac{1}{S} \sum_{s=1}^{S} \hat{\theta}_{T}^{s}(\rho)$, where $\hat{\theta}_{T}^{s}$ is a consistent estimator of the binding function. The indirect estimator of $\rho$ is obtained as the solution of the following minimum distance problem

$$
\hat{\rho}_{T}^{S}=\arg \min _{\rho \in \Re}\left[\hat{\theta}_{T}-\frac{1}{S} \sum_{s=1}^{S} \hat{\theta}_{T}^{s}(\rho)\right]^{\prime} W_{T}\left[\hat{\theta}_{T}-\frac{1}{S} \sum_{s=1}^{S} \hat{\theta}_{T}^{s}(\rho)\right]
$$

For certain structural change tests we will need again to define subsample estimators. They are obtained with the auxiliary model for the first and the second subsamples, namely:

$$
\hat{\theta}_{1 T}^{s}(\rho, \pi)=\arg \min _{\theta_{1} \in \Theta} \frac{1}{[T \pi]} \sum_{t=1}^{[T \pi]} \psi_{1 t}\left(y_{t}^{s}(\rho) \mid Z_{t-1}^{s}(\rho), \theta\right)
$$

and

$$
\hat{\theta}_{2 T}^{s}(\rho, \pi)=\arg \min _{\theta_{2} \in \Theta} \frac{1}{T-[T \pi]} \sum_{t=[T \pi]+1}^{T} \psi_{t}\left(y_{t}^{s}(\rho) \mid Z_{t-1}^{s}(\rho), \theta\right)
$$

The binding function for the two subsamples are then, $\theta_{1}^{*}(\pi)=b_{1}\left(\rho_{1}^{0}, \pi\right)$ and $\theta_{2}^{*}(\pi)=$ $\left.b_{2}\left(\rho_{2}^{0}, \pi\right)\right)$. Therefore the indirect estimators for the first and the second subsamples are obtained by:

$$
\hat{\rho}_{i T}^{S}(\pi)=\arg \min _{\rho_{i} \in \Re}\left[\hat{\theta}_{i T}(\pi)-\frac{1}{S} \sum_{s=1}^{S} \hat{\theta}_{i T}^{s}(\rho, \pi)\right]^{\prime} W_{i T}(\pi)\left[\hat{\theta}_{i T}(\pi)-\frac{1}{S} \sum_{s=1}^{S} \hat{\theta}_{i T}^{s}(\rho, \pi)\right]
$$

for $i=1,2$.

To conclude this section we elaborate on the simulation of processes with structural breaks. Suppose the parameters of interest for the two subsamples are $\rho_{i}$ for $i=1,2$. Then for the first subsample one generates data based on (2.1) and (2.2), modified accordingly, namely $r\left(y_{t}^{s}, y_{t-1}^{s}, x_{t}, u_{t}, \rho_{1}\right)=0$ and $q\left(u_{t}^{s}, u_{t-1}^{s}, \varepsilon_{t}^{s}, \rho_{1}\right)=0$ for $t=1, \cdots,[T \pi]$. This is repeated for the second subsample which covers $t=[T \pi]+1, \cdots, T$ with $\rho_{2}$ as parameter. Hence, one creates a series $\left.\left\{y_{t}^{s}\left(\rho_{1}, \rho_{2}\right)\right\}_{t=1}^{T} \equiv\left(\left\{y_{t}^{s}\left(\rho_{1}\right)\right\}_{t=1}^{[T \pi]}, y_{t}^{s}\left(\rho_{2}\right)\right\}_{t=[T \pi]+1}^{T}\right)$.

\subsubsection{The Efficient Method of Moments estimator}

Gallant and Tauchen (1996) describe the maintained model via a sequence of time-invariant densities $\left\{p_{1}\left(y_{1} \mid \rho\right), p_{t}\left(y_{t} \mid Z_{t-1}, \rho\right)\right\}_{t=1}^{\infty}, \rho \in \Re \subset R^{p}$, whereas the auxiliary model is represented by a sequence of time-invariant densities $\left\{f_{1}\left(y_{1} \mid \theta\right),\left\{f_{t}\left(y_{t} \mid Z_{t-1}, \theta\right)\right\}_{t=1}^{\infty}, \theta \in \Theta \subset R^{q}\right.$. It should be noted that we continue to use $Z_{t-1}$ as the conditional information set. Typically, Gallant and Tauchen consider densities conditional on $\left(y_{t-L}, \cdots, y_{t-1}\right)$. However, in some circumstances $Z_{t-1}$ contains only $x_{t}$, as for instance is the case with reprojection schemes, 
see Gallant and Tauchen (1998). For the sake of simplicity we will keep the conditioning set as $Z_{t-1}$ and it will be obvious from the context what the conditional information set is. The following assumption introduced by Gallant and Tauchen (1996) is used for the validity of the EMM criterion as a specification test for the maintained model.

Assumption 2.2 The maintained model $\left\{p_{1}\left(y_{1} \mid \rho\right), p_{t}\left(y_{t} \mid Z_{t-1}, \rho\right)\right\}_{t=1}^{\infty} \rho \in \Re$ is smoothly embedded within the auxiliary model $\left\{f_{1}\left(y_{1} \mid \theta\right), f_{t}\left(y_{t} \mid Z_{t-1}, \theta\right)\right\}_{t=1}^{\infty} \theta \in \Theta$, i.e. for some open neighborhood $\Re^{0} \rightarrow \Theta$, it is such that: $p_{t}\left(y_{t} \mid Z_{t-1}, \rho\right)=f_{t}\left[y_{t} \mid Z_{t-1}, b(\rho)\right], t=1,2, \ldots$ for every $\rho \in \Re^{0}$ and $p_{1}\left(y_{1} \mid \rho\right)=f_{1}\left(y_{1} \mid b(\rho)\right)$ for every $\rho \in \Re^{0}$.

Under this embedding assumption, the parameters of the auxiliary model $\left(\theta^{*}\right)$ are related to the parameters of the maintained model $\left(\rho^{0}\right)$ according to $\theta^{*}=b\left(\rho^{0}\right)$. Assumption 2.2 is comparable to Assumption 2.1, both play the same role guaranteeing identification of $\rho$ via the auxiliary model. However, Assumption 2.2 is stronger than Assumption 2.1. Indeed, under Assumption 2.2 the E.M.M. estimator is fully efficient.

The EMM estimator is obtained in two steps. The first step is to compute the (quasi) maximum likelihood estimate of the auxiliary model:

$$
\hat{\theta}_{T}=\arg \max _{\theta \in \Theta} \frac{1}{T} \sum_{t=0}^{T} \log \left[f\left(y_{t} \mid Z_{t-1}, \theta\right)\right],
$$

and the corresponding estimate of the information matrix:

$$
I_{T}=\frac{1}{T} \sum_{t=1}^{T}\left[\frac{\partial}{\partial \theta} \log f\left(y_{t} \mid Z_{t-1}, \hat{\theta}_{T}\right)\right]\left[\frac{\partial}{\partial \theta} \log f\left(y_{t} \mid Z_{t-1}, \hat{\theta}_{T}\right)\right]^{\prime} .
$$

In the second step, a vector of moment conditions is constructed using the expectation under the maintained model of the scores from the auxiliary model. The EMM estimator is obtained by minimizing a GMM criterion function formed by the above moment conditions, i.e.,

$$
\hat{\rho}_{T}^{S}=\arg \min _{\rho \in \Re} m_{T}^{S}\left(\rho, \hat{\theta}_{T}\right)^{\prime}\left(I_{T}\right)^{-1} m_{T}^{S}\left(\rho, \hat{\theta}_{T}\right)
$$

where

$$
m_{T}^{S}(\rho, \theta)=\frac{1}{T S} \sum_{t=1}^{T S} \frac{\partial}{\partial \theta} \log \left[f\left(y_{t}^{s}(\rho) \mid Z_{t-1}^{s}(\rho), \theta\right)\right]
$$

and $y_{t}^{s}(\rho), Z_{t-1}^{s}(\rho)_{t=1}^{T S}$ is a long series of realizations simulated from the maintained model with the parameter vector $\rho$. Under suitable regularity conditions discussed in Gallant and Tauchen (1996) and Assumption 2.2, we have $\sqrt{T}\left(\hat{\theta}_{T}-\theta_{0}\right) \stackrel{d}{\rightarrow} N\left\{0, I^{-1}\right\}$ and, $\sqrt{T}\left(\hat{\rho_{T}}-\rho^{0}\right) \stackrel{d}{\rightarrow}$ $N\left\{0,\left[M_{\rho}^{\prime} I^{-1} M_{\rho}\right]^{-1}\right\}$ where $M_{\rho}=\left(\partial / \partial \rho^{\prime}\right) m\left(\rho^{0}, \theta_{0}\right)$ and $I$ is the outer product of scores, as suggested by the estimator in (2.17). All these results apply to the case where the number 
of simulations goes to infinity. In the case of possible structural changes with unknown breakpoint, theoretical results based of the number of simulations equal to infinity are not so appealing as the computational cost involved can be prohibitively high. For this reason, the asymptotic results need to be modified to account for a finite number of simulations. When $S$ is finite, the randomness of the EMM estimator $\hat{\rho}_{T}$ will not only depend on the randomness of $\hat{\theta}_{T}$ but also on the randomness of the moment conditions due to a finite length of series simulated from the structural model. Therefore the asymptotic variancecovariance matrix in equation (2.17) is scaled by $(1+1 / S)$ using arguments similar to Duffie and Singleton (1993).

To conclude this section we present partial sample estimators which appear in certain tests for structural change. The unrestricted EMM estimator for the subsamples are defined as:

$$
\hat{\rho}_{i T}^{S}(\pi)=\arg \min _{\rho_{i} \in \Re} m_{i}^{\prime}\left(\rho, \hat{\theta}_{i T}(\pi)\right)\left(I_{i T}\right)^{-1} m_{i}\left(\rho, \hat{\theta}_{i T}(\pi)\right)
$$

where $\rho_{i} \in \Re \subset R^{p}, I_{i T}$ is the estimator of the matrix $I$ for the $i^{t h}$ subsample and:

$$
\begin{gathered}
\left.m_{1}\left(\rho, \hat{\theta}_{1 T}(\pi)\right)=\frac{1}{[T S \pi]} \sum_{t=1}^{[T S \pi]} \frac{\partial}{\partial \theta} \log f\left(y_{t}^{s}(\rho) \mid Z_{t-1}^{s}(\rho), \hat{\theta}_{1}(\pi)\right)\right] . \\
m_{2}\left(\rho, \hat{\theta}_{2 T}(\pi)\right)=\frac{1}{T S-[T S \pi]} \sum_{t=[T S \pi]+1}^{T S} \frac{\partial}{\partial \theta} \log f\left[y_{t}^{s}(\rho) \mid Z_{t-1}^{s}(\rho), \hat{\theta}_{2}(\pi)\right] .
\end{gathered}
$$

The simulation of processes when a break is present can be characterized by the following sequence of densities $\left\{p_{1}\left(y_{1} \mid \rho_{1}\right), p_{t}\left(y_{t} \mid Z_{t-1}, \rho_{1}\right)\right\}_{t=1}^{[T \pi]}$, for the first subsample and

$\left\{p_{t}\left(y_{t} \mid Z_{t-1}, \rho_{2}\right)\right\}_{t=[T \pi]+1}^{T}$, for the second. It is important to note that the simulated path length is function of the fraction of the sample $(\pi)$. This point is crucial. Indeed, asymptotic distribution of several structural change tests could depend on the nuisance parameter $S$ and hence the critical values depend on $\mathrm{S}$, in the case where the simulated path length is not split according to the presumed breakpoint $\pi$. Section 3.2 will examine this problem.

\section{GMM-like Tests for Structural change with unknown breakpoint}

The purpose of this section is to generalize GMM-based tests for structural change presented by Andrews (1993), Andrews and Ploberger (1994), Sowell (1996a,b) and Ghysels, Guay and Hall (1997). A variety of tests were proposed ranging from (optimal) Wald, LM and LR-type tests to predictive tests with unknown breakpoint. In this section we deal with the issues 
posed by procedures involving two models, an auxiliary one and a model of interest. We noted that the role played by both models poses challenges and provides new opportunities for hypothesis testing. Here we only deal with the usual Wald, LM and LR-type and predictive tests. In the next section we cover tests which are specifically designed for the dual model setup. We cover tests based on ALS, Indirect Inference and EMM estimators. One of the first issues to resolve is to clearly define the null hypothesis of interest in tests for structural change analysis. The analysis in the first subsection involves the ALS since it allows us again to focus directly on the key issues of hypotheses and test statistics. The added complication of simulation uncertainty is considered in the second subsection.

\subsection{Tests for Asymptotic Least Squares}

The purpose of this section is twofold: (1) clearly spell out the null hypotheses involved in tests for structural change when an auxiliary model is present and (2) adapt the usual Wald, LM and LR-type and predictive tests for such situations. A subsection is devoted to each of the two issues.

\subsubsection{The Null Hypotheses}

The null hypothesis of interest is:

$$
H_{0}^{\rho}: \rho_{t}=\rho_{0} \quad \forall t=1, \ldots, T .
$$

The fact that we estimate the parameter vector $\rho$ indirectly via an auxiliary model implies that we also should consider the null hypothesis:

$$
H_{0}^{\theta}: \theta_{t}=\theta_{0} \quad \forall t=1, \ldots, T
$$

The null hypotheses (3.1) and (3.2), while related, are obviously not identical. Accepting $H_{0}^{\theta}$ implies that there is no structural change for $\rho$ because of the identification Assumption 2.1 for the binding function. Rejecting $H_{0}^{\theta}$ does not necessarily imply that $H_{0}^{\rho}$ is violated since the dimension of $\theta$ is equal or greater than the dimension of $\rho$. To unravel whether the rejection of $H_{0}^{\theta}$ is due to a structural change of the overidentifying restrictions, one can follow the approach of Sowell (1996a) and characterize via projection the subspace which identifies $\rho$. Such projection can distinguish structural change of the structural parameters from breaks in the overidentifying restrictions. This distinction becomes even more interesting when we allow for partial structural change, i.e. consider subvectors of $\rho$. In particular, in the 
context of semiparametric indirect inference, following Broze et al. (1998), this may involve a subvector of nuissance parameters $\rho^{2}$ for which structural change may be more tolerated.

To elaborate further on the distinction between the null hypotheses (3.1) and (3.2), and in particular the interpretation of rejecting the null hypotheses, we consider a sequence of local of alternatives:

$$
\theta_{t, T}=\theta^{*}+h\left(\eta, s, \frac{t}{T}\right) / \sqrt{T}
$$

where $h(\eta, s, \pi)$, for $\pi \in[0,1]$, is a q-dimensional function which can be expressed as the uniform limit of step functions, $\eta \in R^{i}, s \in R^{j}$ such that $0<s_{1}<s_{2}<\ldots<s_{j}<1$ and $\theta^{*}$ is in the interior of $\Theta$. The function $h(\cdot)$ allows for a wide range of alternative hypotheses (see Sowell (1996a)). The parameter $s$ locates structural changes as a fraction of the sample size and the vector $\eta$ defines the local alternatives. To simplify the notation $h\left(\eta, s, \frac{t}{T}\right)$ will be noted $h(s)$. The following theorem provides the asymptotic distribution for the optimally weighted $g(\cdot)$ for both subsamples, using $W_{T}=\Omega_{T}^{-1}$, where $\Omega_{T}$ is the full sample estimator of the optimal weighting matrix $\Omega$ which is defined in Appendix E:

Theorem 3.1 Under Assumptions A.1, A.2, B.1 and sequence of local alternatives (3.3), we have:

$$
\begin{aligned}
\pi \sqrt{T} \Omega_{T}^{-1 / 2} g\left(\hat{\theta}_{1 T}(\pi), \hat{\rho}_{T}\right) \Rightarrow & -B(\pi)-\Omega^{-1 / 2} G_{\theta} H(\pi)+ \\
& \pi \Omega^{-1 / 2} G_{\rho}\left(G_{\rho}^{\prime} \Omega^{-1} G_{\rho}\right)^{-1} G_{\rho}^{\prime} \Omega^{-1 / 2}\left[B(1)-\Omega^{-1 / 2} G_{\theta} H(1)\right] . \\
(1-\pi) \sqrt{T} \Omega_{T}^{-1 / 2} g\left(\hat{\theta}_{2 T}(\pi), \hat{\rho}_{T}\right) \Rightarrow & -\left[B(1)-B(\pi)-\Omega^{-1 / 2} G_{\theta}(H(1)-H(\pi))\right]+ \\
& (1-\pi) \Omega^{-1 / 2} G_{\rho}\left(G_{\rho}^{\prime} \Omega^{-1} G_{\rho}\right)^{-1} G_{\rho}^{\prime} \Omega^{-1 / 2}\left[B(1)-\Omega^{-1 / 2} G_{\theta} H(1)\right] .
\end{aligned}
$$

where $H(\pi)=\int_{0}^{\pi} h(\eta, s, u) d u, B(\pi)$ is a q-dimensional vectors of independent Brownian motions and $G_{\rho}, G_{\theta}$ are defined in B.1.

Proof: See Appendix E

Under the null hypothesis (3.2), a version of Corollary 1 of Sowell(1996a) holds, namely there exists an orthonormal matrix $\mathrm{C}$ such that

$$
\pi C \sqrt{T} \Omega_{T}^{-1 / 2} g\left(\hat{\theta}_{1 T}(\pi), \hat{\rho}_{T}\right) \Rightarrow\left[\begin{array}{c}
-B B_{p}(\pi) \\
-B_{G-p}(\pi)
\end{array}\right] .
$$


where $B B_{p}(\pi)$ is a $p$-dimensional Brownian bridge, $B(\pi)$ is a $G$-p-dimensional Brownian motion, $C$ is such that $\Omega^{-1 / 2} G_{\rho}\left(G_{\rho}^{\prime} \Omega^{-1} G_{\rho}\right)^{-1} G_{\rho}^{\prime} \Omega^{-1 / 2}=C^{\prime} \Lambda C, C C^{\prime}=I$ and

$$
\Lambda=\left[\begin{array}{cc}
I_{p} & 0_{p \times(G-p)} \\
0_{(G-p) \times p} & 0_{(G-p) \times(G-p)}
\end{array}\right] .
$$

For the function $g(\cdot)$ evaluated at the estimator obtained with the second subsample, we have

$$
(1-\pi) C \sqrt{T} \Omega_{T}^{-1 / 2} g\left(\hat{\theta}_{2 T}(\pi), \hat{\rho}_{T}\right) \Rightarrow\left[\begin{array}{c}
B B_{p}(\pi) \\
-B_{G-p}^{*}(\pi)
\end{array}\right]
$$

where $B B_{q}(\pi)$ is defined above and $B_{G-p}^{*}(\pi)=B_{G-p}(1)-B_{G-p}(\pi)$.

As shown by Sowell (1996b), structural change tests can be constructed in projecting on the appropriate subspace. The limiting stochastic processes in (3.4) and (3.5) are equivalent to the limiting stochastic processes for the GMM estimator in Sowell or those obtained for the Simulated Method of Moments estimator in Ghysels and Guay (2001). Under the null hypotheses (3.1) and (3.2), the results in (3.4) and (3.5) show that the limiting continuous stochastic processes are linear combinations of $p$ Brownian bridges, one for each parameter estimated, and $G-p$ Brownian motions, spanning the space of overidentifying restrictions, where $G$ is the dimension of $g(\cdot)$.

We can refine now the null hypothesis (3.1). In particular, following Hall and Sen (1999) we consider the generic null, for the case of a single breakpoint, which seperates the identifying restrictions across the two subsamples:

$$
H_{0}^{I \rho}(\pi)= \begin{cases}P_{G}^{\prime} \Omega^{-1 / 2} g\left(\theta^{*}(\rho), \rho^{0}\right)=0 & \forall t=1, \ldots,[\pi T] \\ P_{G} \Omega^{-1 / 2} g\left(\theta^{*}(\rho), \rho^{0}\right)=0 & \forall t=[\pi T]+1, \ldots, T\end{cases}
$$

where $P_{G}=\Omega^{-1 / 2} G_{\rho}\left(G_{\rho}^{\prime} \Omega^{-1} G_{\rho}\right)^{-1} G_{\rho}^{\prime} \Omega^{-1 / 2}$. Moreover, the overidentifying restrictions are stable if they hold before and after the breakpoint. This is formally stated as $H_{0}^{O-g}(\pi)=$ $H_{0}^{O-g 1}(\pi) \cap H_{0}^{O-g 2}(\pi)$ with:

$$
\begin{array}{ll}
H_{0}^{O-g 1}(\pi):\left(I_{G}-P_{G 1}(\pi)\right) \Omega_{1}^{-1 / 2}(\pi) g\left(\theta^{*}(\rho), \rho^{0}\right)=0 & \forall t=1, \ldots,[\pi T] \\
H_{0}^{O-g 2}(\pi):\left(I_{G}-P_{G 2}(\pi)\right) \Omega_{2}^{-1 / 2}(\pi) g\left(\theta^{*}(\rho), \rho^{0}\right)=0 & \forall t=[\pi T]+1, \ldots, T
\end{array}
$$

where $P_{G i}(\pi)$ and $\Omega_{i}(\pi)$ are the subsample equivalents of $P_{G}$ and $\Omega$ respectively for $i=$ 1,2. By projection the decomposition appearing in (3.4) and (3.5), it is clear that instability must be reflected in a violation of at least one of the three hypotheses: $H_{0}^{I \rho}(\pi), H_{0}^{O-g 1}(\pi)$, or 
$H_{0}^{O-g 2}(\pi)$. It is only the former of those three which corresponds to the null hypothesis (3.1). Violation of $H_{0}^{O-g 1}(\pi)$, or $H_{0}^{O-g 2}(\pi)$ mean that there are reasons to reject the null hypothesis $H_{0}^{\theta}$ in (3.2), but still accept $H_{0}^{\rho}$ in (3.1). Various tests can be constructed with local power properties against any particular one of these three null hypotheses (and typically no power against the others).

To conclude we need to discuss the implication of various structural change tests in presence of auxiliary models. The decomposition of the hypothesis (and associated tests) into $H_{0}^{I \rho}(\pi), H_{0}^{O-g 1}(\pi)$, or $H_{0}^{O-g 2}(\pi)$ has different implications for the structural model. The auxiliary model can be viewed as a window through which information is obtained about the structural model. Consequently, structural change can only be assessed via the information about the structural model revealed by the auxiliary model. For example, Guay and Renault (2001) examine indirect encompassing when both models are misspecified and estimated by auxiliary models. In the first step of their proposed procedure, the auxiliary model is used only to obtain consistent estimators of the parameters of structural models. Structural parameter instability detected through the intermediary of the auxiliary model is crucial for the consistency of the procedure. However, instability of the overidentifying restrictions (of the auxiliary model) without change of the structural parameters is innocuous.

\subsubsection{Test statistics}

A structural change test is obtained for the vector of parameters $\rho$ when the function $S_{T}^{-1 / 2} g(\cdot)$ is projected on the subspace identifying the parameters with the first subsample estimator $\hat{\theta}_{1 T}$. This statistic is

$$
\begin{aligned}
Q_{1 T}(\pi)= & T \pi^{2} g\left(\hat{\theta}_{1 T}(\pi), \hat{\rho}_{T}\right)^{\prime} \Omega_{T}^{-1 / 2}\left(\Omega_{T}^{-1 / 2} G_{\rho, T}\left(G_{\rho, T}^{\prime} \Omega_{T}^{-1} G_{\rho, T}\right)^{-1} G_{\rho, T}^{\prime} \Omega_{T}^{-1 / 2}\right) \\
& \Omega_{T}^{-1 / 2} g\left(\hat{\theta}_{1 T}(\pi), \hat{\rho}_{T}\right) .
\end{aligned}
$$

The statistic with the estimator of the second subsample is:

$$
\begin{aligned}
Q_{2 T}(\pi)= & T(1-\pi)^{2} g\left(\hat{\theta}_{2 T}(\pi), \hat{\rho}_{T}\right)^{\prime} \Omega_{T}^{-1 / 2}\left(\Omega_{T}^{-1 / 2} G_{\rho, T}\left(G_{\rho, T}^{\prime} \Omega_{T}^{-1} G_{\rho, T}\right)^{-1} G_{\rho, T}^{\prime} \Omega_{T}^{-1 / 2}\right) \\
& \Omega_{T}^{-1 / 2} g\left(\hat{\theta}_{2 T}(\pi), \hat{\rho}_{T}\right) .
\end{aligned}
$$

A structural change test for overidentifying restrictions is obtained with the statistic which consists of projecting the function $\Omega_{T}^{-1 / 2} g(\cdot)$ on the subspace orthogonal to the subspace identifying the parameters. For example, the statistic with the first subsample estimator is

$$
\begin{aligned}
Q_{1 T}^{0}(\pi)= & T(\pi)^{2} g\left(\hat{\theta}_{1 T}(\pi), \hat{\rho}_{T}\right)^{\prime} \Omega_{T}^{-1 / 2}\left(I-\Omega_{T}^{-1 / 2} G_{\rho, T}\left(G_{\rho, T}^{\prime} \Omega_{T}^{-1} G_{\rho, T}\right)^{-1} G_{\rho, T}^{\prime} \Omega_{T}^{-1 / 2}\right) \\
& \Omega_{T}^{-1 / 2} g\left(\hat{\theta}_{1 T}(\pi), \hat{\rho}_{T}\right)
\end{aligned}
$$


In the case of unknown breakpoint, statistics can be constructed by mapping on $\pi \in$ П. Andrews and Ploberger (1994) in the context of maximum likelihood estimation and Sowell (1996a,b) for GMM estimation derive optimal tests which are characterized by an average exponential mapping. In the case of a one time structural break alternative and a particular integral weight functions for $h(\cdot)$ in $(3.3)$, the tests with the greatest weighted average asymptotic power have the following form for structural parameter instability

$$
\operatorname{Exp}-Q_{i T}=(1+c)^{(-p / 2)} \int \exp \left(\frac{1}{2} \frac{c}{1+c} Q_{i T}(\pi)\right) d R(\pi)
$$

where $R(\pi)$ is the weight function over the set of possible breakpoints $\Pi$. The parameter $\mathrm{c}$ controls the distance of the alternative. For close alternatives, the asymptotic test with the greatest weighted average power is an average over $\pi \in \Pi$ and has the form: $\int Q_{i T}(\pi) d R(\pi)$. For a distant alternative, the functional is: $\log \int \exp \left(\frac{1}{2} Q_{i T}(\pi)\right) d R(\pi)$. The supremum form $\sup _{\pi \in \Pi} Q_{i t}(\pi)$ often used in the litterature corresponds to the case where $c /(1+c) \rightarrow \infty$. The LM (or $L M_{T}(\pi)$ for given $\pi$ ) test statistic of structural change corresponds to the case where $R(\pi)=1 /(\pi(1-\pi)) d \pi$. A Wald $\left(\operatorname{Wald}_{T}(\pi)\right)$ and a LR-type $\left(\operatorname{LR}_{T}(\pi)\right)$ test statistics can be constructed as usual with the restricted and unrestricted ALS estimators. Following Andrews (1993), we can show that $\operatorname{Wald}_{T}(\pi)=L M_{T}(\pi)+o_{p}(1)$ and $L R_{T}(\pi)=L M_{T}(\pi)+o_{p}(1)$.

The following proposition gives the asymptotic distribution for the exponential mapping for $Q_{i T}$ when $Q_{i T}$ corresponds to the Wald, LM and LR ratio-type tests.

Proposition 3.1 Under the null hypothesis $H_{0}$ in (3.1) and Assumptions A.1, A.2, B.1, the following processes indexed by $\pi$ for a given set $\Pi$ whose closure lies in $(0,1)$ satisfy:

$\sup Q_{i T} \Rightarrow \sup _{\pi \in \Pi} Q_{p}(\pi), \quad$ ave $Q_{i T} \Rightarrow \int_{\Pi} Q_{p}(\pi) d R(\pi), \quad \exp Q_{i T} \Rightarrow \log \left(\int_{\Pi} \exp \left[\frac{1}{2} Q_{p}(\pi)\right] d R(\pi)\right)$, with

$$
Q_{p}(\pi)=B B_{p}(\pi)^{\prime} B B_{p}(\pi)
$$

for $i=1,2$.

This result is obtained through the application of the continuous mapping theorem (see Pollard (1984)). The asymptotic distribution is a quadratic form of weighted Brownian bridge such as when the breakpoint is known the asymptotic distribution is a chi-square with a degree of freedom equal to the dimension of the structural vector parameters.

The next proposition gives the asymptotic distribution for the exponential mapping for $Q_{i T}^{0}$ when $Q_{i T}^{0}$ is the statistic for the structural change in overidentifying restrictions corresponding to the null $H_{0}^{O-g i}(\pi)$. 
Proposition 3.2 Under the null hypothesis of no structural change for the over-identifying restrictions and Assumptions A.1, A.2, B.1, the following processes indexed by $\pi$ for a given set $\Pi$ whose closure lies in $(0,1)$ satisfy:

$$
\begin{gathered}
\sup Q_{i T}^{0} \Rightarrow \sup _{\pi \in \Pi} Q_{i, G-p}(\pi), \\
\operatorname{ave}_{i T}^{0} \Rightarrow \int_{\Pi} Q_{i, G-p}(\pi) d R(\pi), \\
\exp Q_{i T}^{0} \Rightarrow \log \left(\int_{\Pi} \exp \left[\frac{1}{2} Q_{i, G-p}(\pi)\right] d R(\pi)\right),
\end{gathered}
$$

with $Q_{1, G-p}(\pi)=B_{G-p}(\pi)^{\prime} B_{G-p}(\pi)$ and $Q_{2, G-p}(\pi)=B_{G-p}^{*}(\pi)^{\prime} B_{G-p}^{*}(\pi)$, where $B_{G-p}(\pi)$ is a $G-p$-dimensional vector of independent Brownian motion, $B_{G-p}^{*}(\pi)=B_{G-p}(1)-B_{G-p}(\pi)$ and $i=1,2$.

The asymptotic distribution is a quadratic form of Brownian motion such as when the breakpoint is known the asymptotic distribution is a chi-square with a degree of freedom equal to $G-p$. Predictive tests, discussed in Ghysels, Guay and Hall (1997) and Guay (1996), can also be constructed and the asymptotic distribution of those tests can be easily obtained from Theorem 3.1.

\subsection{Structural Change for Indirect Inference and EMM}

The analysis in Section 3.1 involves the Asymptotic Least Squares estimator of Gouriéroux, Monfort and Trognon (1985), a procedure which we have chosen to discuss first as it features the estimation of $\rho$ through an auxiliary model parameterized by $\theta$. We now turn our attention to procedures with similar features, but which require simulations to obtain the binding function appearing in (2.5). Sidestepping simulation uncertainty allowed us to focus exclusively on the key issue of testing for structural change when an auxiliary model is present. The results in Ghysels and Guay (2001) may help us to understand the effect of simulation uncertainty on tests for structural change. They propose a set of tests for structural change in models estimates via Simulated Method of Moments (see Duffie and Singleton (1993)) and show that the number of simulations does not affect the asymptotic distribution nor the asymptotic local power of tests for structural change. Hence, the asymptotic results obtained for GMM-based tests are also valid for SMM-based procedures. The intuition for this result is that in the case of tests for structural change one compares parameter estimates that are subject to the same simulation uncertainty (unlike tests of a fixed hypothesis where the distance of the estimates to the null depends on the simulation uncertainty). The result 
in Ghysels and Guay (2001) critically depends on the choice of the weighting matrix and a Monte Carlo investigation also reveals that simulation uncertainty does affect the finite sample properties of tests. Nontheless, it is also shown that a relatively small number of simulations suffices to obtain tests with desirable small sample size and power properties.

The purpose of this section is to extend the results of Ghysels and Guay (2001). In particular, it will be shown that for both the I.I. and EMM estimators, the simulation uncertainty does not affect the asymptotic distribution of tests for structural stability. Hence, there is an asymptotic analogue between ALS-based tests and I.I. or EMM-based procedures. We begin with the Indirect Inference procedure to show that this is indeed the case.

Theorem 3.2 For the full and the partial sample indirect inference estimators appearing in (2.13), (2.14), under Assumptions A.2 and C.1 and (3.3), we have

$$
\begin{aligned}
\pi \sqrt{T} \Omega_{T}^{-1 / 2}\left[\hat{\theta}_{1 T}(\pi)-\frac{1}{S} \sum_{s=1}^{S} \theta_{1 T}^{s}\left(\rho_{T}^{s}, \pi\right)\right] \Rightarrow & -\left[B(\pi)-\frac{1}{S} \sum_{s=1}^{S} B^{s}(\pi)-\Omega^{-1 / 2} H(\pi)\right] \\
& +\pi \Omega^{-1 / 2} b_{\rho}\left[b_{\rho}^{\prime} \Omega^{-1} b_{\rho}\right]^{-1} b_{\rho}^{\prime} \Omega^{-1 / 2} \times \\
& {\left[B(1)-\frac{1}{S} \sum_{s=1}^{S} B^{s}(1)-\Omega^{-1 / 2} H(1)\right] }
\end{aligned}
$$

and for the second subsample

$$
\begin{array}{r}
(1-\pi) \sqrt{T} \Omega_{T}^{-1 / 2}\left[\hat{\theta}_{2 T}(\pi)-\frac{1}{S} \sum_{s=1}^{S} \theta_{2 T}^{s}\left(\rho_{T}^{s}, \pi\right)\right] \Rightarrow-\left[B(1)-B(\pi)-\frac{1}{S} \sum_{s=1}^{S}\left(B(1)^{s}-B(\pi)^{s}\right)\right] \\
+\Omega^{-1 / 2}(H(1)-H(\pi))+(1-\pi) \Omega^{-1 / 2} b_{\rho}\left[b_{\rho}^{\prime} \Omega^{-1} b_{\rho}\right]^{-1} \times \\
b_{\rho}^{\prime} \Omega^{-1 / 2}\left[B(1)-\frac{1}{S} \sum_{s=1}^{S} B^{s}(1)-\Omega^{-1 / 2} H(1)\right]
\end{array}
$$

where $H(\pi)=\int_{0}^{\pi} h(\eta, s, u) d u$ and $B(\pi)$ and $B^{s}(\pi)$ are two $q$-dimensional vectors of mutually independent Brownian motions and $\Omega_{T}=J_{T}^{-1} I_{T} J_{T}^{-1}$.

Proof: See Appendix E

Under the null hypothesis, by replacing $\Omega_{T}$ by $\tilde{\Omega}_{T}=\left(1+\frac{1}{S}\right) \Omega_{T}$ in the expressions of Theorem 3.2, a version of Corollary 1 of Sowell(1996a) can be easily shown such as

$$
\pi C \sqrt{T} \tilde{\Omega}_{T}^{-1 / 2}\left[\hat{\theta}_{1 T}(\pi)-\frac{1}{S} \sum_{s=1}^{S} \theta_{1 T}^{s}\left(\rho_{T}^{s}, Z_{0}^{s}, \pi\right)\right] \Rightarrow\left[\begin{array}{c}
-B B_{p}(\pi) \\
-B_{q-p}(\pi)
\end{array}\right] .
$$

For the second subsample, we have

$$
(1-\pi) C \sqrt{T} \tilde{\Omega}_{T}^{-1 / 2}\left[\hat{\theta}_{2 T}(\pi)-\frac{1}{S} \sum_{s=1}^{S} \theta_{2 T}^{s}\left(\rho_{T}^{s}, Z_{0}^{s}, \pi\right)\right] \Rightarrow\left[\begin{array}{c}
B B_{p}(\pi) \\
-B_{q-p}^{*}(\pi)
\end{array}\right] .
$$


To obtained these results, we have just to see that

$$
\left(1+\frac{1}{S}\right)^{-1 / 2}\left[B(\pi)-\frac{1}{S} \sum_{s=1}^{S} B^{s}(\pi)\right]
$$

is a $q$-dimensional vector of standard Brownian motion. As shown in Section 3.3, structural change tests can be constructed by projection on the appropriate subspace. A structural change test is obtained for the vector of parameters $\rho$ when the difference between the estimator obtained with the auxiliary model for the data and the average estimators obtained with simulated paths is projected on the subspace identifying the parameters for the first or the second subsample. This statistic is

$T \pi^{2}\left[\hat{\theta}_{i T}(\pi)-\frac{1}{S} \sum_{s=1}^{S} \theta_{i T}^{s}\left(\rho_{T}^{s}\right)\right]^{\prime} \tilde{\Omega}_{T}^{-1 / 2}\left(\tilde{\Omega}_{T}^{-1 / 2} b_{\rho}\left[b_{\rho}^{\prime} \tilde{\Omega}_{T}^{-1} b_{\rho}\right]^{-1} b_{\rho}^{\prime} \tilde{\Omega}_{T}^{-1 / 2}\right) \tilde{\Omega}_{T}^{-1 / 2}\left[\hat{\theta}_{i T}(\pi)-\frac{1}{S} \sum_{s=1}^{S} \theta_{i T}^{s}\left(\rho_{T}^{s}\right)\right]$

where $i=1,2$ depending on the subsample. A structural change tests for overidentifying restrictions is obtained by projecting the same function on the subspace orthogonal to the subspace identifying the parameters. The resulting statistic is:

$$
\begin{array}{r}
T(1-\pi)^{2}\left[\hat{\theta}_{i T}(\pi)-\frac{1}{S} \sum_{s=1}^{S} \theta_{i T}^{s}\left(\rho_{T}^{s}\right)\right]^{\prime} \tilde{\Omega}_{T}^{-1 / 2}\left(I-\tilde{\Omega}_{T}^{-1 / 2} b_{\rho}\left[b_{\rho}^{\prime} \tilde{\Omega}_{T}^{-1} b_{\rho}\right]^{-1} b_{\rho}^{\prime} \tilde{\Omega}_{T}^{-1 / 2}\right) \\
\tilde{\Omega}_{T}^{-1 / 2}\left[\hat{\theta}_{i T}(\pi)-\frac{1}{S} \sum_{s=1}^{S} \theta_{i T}^{s}\left(\rho_{T}^{s}\right)\right]
\end{array}
$$

The asymptotic distribution of the exponential mappings of these statistics is given in Proposition 3.1 for the parameter stability and in Proposition 3.2 for stability of overidentifying restrictions.

Theorem 3.2 shows that simulation uncertainty does not affect the asymptotic distribution of tests for structural stability. Hence, the implication of structural change detected in the auxiliary model has the same interpretation as in the ALS case. In Section 3.1.1 it was noted that the importance of instability in the auxiliary model for the structural model depends on which hypotheses is violated, namely $H_{0}^{I \rho}(\pi), H_{0}^{O-g 1}(\pi)$, or $H_{0}^{O-g 2}(\pi)$. An interesting case to examine can be found in Dridi and Renault (2001) who develop a generalization of Indirect Inference to semi-parametric settings. Their approach produces a theory of robust estimation despite misspecifications of the structural model. Suppose economic theory only provides information about a subvector of parameters of interest $\rho^{1}$ (in our notation) and direct estimation of the structural model can not be performed so that the econometrician relies on Indirect Inference. To simulated the structural model, an additional nuisance parameter vector $\rho^{2}$ is required. Since we are only interested in a consistent estimator for $\rho^{1}$, 
the importance of finding structural change in the auxiliary model depends on the impact of instability on the parameter vector of interest. In particular, instability for the nuisance parameters $\rho^{2}$ or for overidentifying restrictions without affecting stability of the parameter vector of interest have no impact on the consistency of the semi-parametric Indirect Inference estimator. Structural change tests must therefore focus on the parameter vector of interest $\rho^{1}$. With our results, partial structural stability tests for this parameter vector of interest can be constructed and the asymptotic distribution of exponential mappings is given in Section 3.1 .2 .

Next, we turn to the EMM estimator, in particular:

Theorem 3.3 For the partial sample Efficient Method of Moments estimators appearing in (2.18), (2.21), (2.22), under Assumptions A.2 and D.1, we have

$$
\begin{aligned}
\pi \sqrt{T} I_{T}^{-1 / 2} m_{1}\left(\hat{\rho}_{T}^{S}, \hat{\theta}_{1 T}(\pi)\right) \Rightarrow & -\left[B(\pi)-\frac{1}{\sqrt{S}} B^{S}(\pi)+I^{-1 / 2} J H(\pi)\right] \\
& +\pi I^{-1 / 2} M_{\rho}\left[M_{\rho}^{\prime} I^{-1} M_{\rho}\right]^{-1} M_{\rho}^{\prime} I^{-1 / 2}\left[B(1)-\frac{1}{\sqrt{S}} B^{s}(1)+I^{-1 / 2} J H(1)\right]
\end{aligned}
$$

and for the second subsample

$$
\begin{aligned}
(1-\pi) \sqrt{T} I_{T}^{-1 / 2} m_{2}\left(\hat{\rho}_{T}^{S}, \hat{\theta}_{2 T}(\pi)\right) \Rightarrow & -\left[B(1)-B(\pi)-\frac{1}{\sqrt{S}}\left(B(1)^{S}-B(\pi)^{s}\right)+I^{-1 / 2} J(H(1)-H(\pi))\right] \\
& +\pi I^{-1 / 2} M_{\rho}\left[M_{\rho}^{\prime} I^{-1} M_{\rho}\right]^{-1} M_{\rho}^{\prime} I^{-1 / 2}\left[B(1)-\frac{1}{\sqrt{S}} B^{s}(1)+I^{-1 / 2} J H(1)\right]
\end{aligned}
$$

where $H(\pi)=\int_{0}^{\pi} h(\eta, s, u) d u$ and $B(\pi)$ and $B^{s}(\pi)$ are two $q$-dimensional vectors of mutually independent Brownian motions.

\section{Proof: See Appendix E}

Structural change tests can be constructed by replacing $I_{T}$ by $\tilde{I}_{T}=\left(1+\frac{1}{S}\right) I_{T}$ as shown in the previous sections. The asymptotic distribution of the tests is the same since $(1+$ $1 / S)^{-1 / 2}\left[B(\pi)-\frac{1}{\sqrt{S}} B^{s}(\pi)\right]$ is a $q$-dimensional vector of Brownian motions. The asymptotic distributions are given in Proposition 3.1 for the parameter stability and in Proposition 3.2 for stability of overidentifying restrictions.

van der Sluis (1998) proposes similar structural change tests for EMM. However, in contrast to our strategy, the length of the simulated series used to construct the structural change tests in van der Sluis (1998) is the same for the estimation of the full sample estimator of $\rho$ and for the evaluation of the moment restrictions with the unrestricted estimators $\theta_{i T}(\pi)$. Such a strategy has an important impact on the asymptotic distribution as will be shown in the remaining of this section. Suppose that the length of the simulated series is equal to 
$T S$. The statistic proposes by van der Sluis is based on the following moment restrictions:

$$
\frac{1}{T S} \sum_{t=1}^{T S} m\left(\hat{\rho}_{T}^{S}, \hat{\theta}_{i T}(\pi)\right)
$$

for $i=1,2$. For the case where the moment restrictions are evaluated at $\hat{\theta}_{1 T}(\pi)$ which is also obtained with a simulated path equal to $T S$, we can show the following result under the null:

$$
\begin{aligned}
\sqrt{T} I_{T}^{-1 / 2} \frac{1}{[T S]} \sum_{t=1}^{[T S]} m\left(\hat{\rho}_{T}^{S}, \hat{\theta}_{1 T}(\pi)\right) \Rightarrow & -\left[\frac{B(\pi)}{\pi}-\frac{1}{\sqrt{S}} B^{S}(1)\right] \\
& +I^{-1 / 2} M_{\rho}\left[M_{\rho}^{\prime} I^{-1} M_{\rho}\right]^{-1} M_{\rho}^{\prime} I^{-1 / 2}\left[B(1)-\frac{1}{\sqrt{S}} B^{S}(1)\right] .
\end{aligned}
$$

The LM structural change statistic is constructed by projecting the above moment restrictions on the subspace identifying the parameters. Such a statistic has the usual asymptotic distribution (see Proposition 3.1). This result holds because the nuisance term introduced by simulation $(1 / \sqrt{S}) B^{S}(1)$ cancels out. However, the asymptotic distribution of a structural change test for overidentifying restrictions constructed by projection on the subspace orthogonal to the subspace identifying the parameters is not the same as given in Proposition 3.2. In this case, one can show that the asymptotic distribution is given by the following process:

$$
B_{G-p}^{S}(1)^{\prime} B_{G-p}^{S}(1)+B_{G-p}(\pi)^{\prime} B_{G-p}(\pi) .
$$

This expression contains a nuisance parameter that depends on the length of the simulated path, and hence the critical values depend on $S$. Consequently, the statistics in van der Sluis (1998) are valid only in the case where $S$ equals infinity, or would require critical values that need to be computed for various values of $S$ (the applications in van der Sluis (1998) use $S=3$ with $S=\infty$ critical values).

\section{Tests Exploiting Auxiliary Models}

Thus far we examined a set of tests which were introduced to the literature in the context of GMM and SMM estimation and have their roots in the earlier literature (see e.g. Andrews (1993) for references). We studied the consequences of having estimation and inference via auxiliary models. The purpose of this section is to present statistics which are designed to tests for structural breaks and take advantage of the dual model setup. We cover two types of tests, a first class relates to recent work of Liu and Zhang (1998) on diagnostic testing of 
EMM score generators which we show are implicitly tests for structural change. The second class is based on the simulated score principle.

\subsection{Liu and Zhang Tests}

The results obtained in Appendix E allow us to examine the specification test in the Efficient Method of Moments framework proposes by Liu and Zhang (1998). This test is a measure of the overall goodness of fit of the auxiliary model. The zeta statistic introduced by Liu and Zhang is defined as follows:

$$
\zeta_{T}=\frac{12}{T^{3}}\left(\sum_{i=1}^{T} \sum_{t=1}^{i} s_{t}^{\prime}\left(\hat{\theta}_{T}\right)\right)\left(I_{T}\right)^{-1}\left(\sum_{i=1}^{T} \sum_{t=1}^{i} s_{t}\left(\hat{\theta}_{T}\right)\right)
$$

where $s_{t}\left(\theta_{T}\right)=\partial / \partial \theta \log f\left(y_{t} \mid x_{t-1}, \theta_{T}\right)$. We will show that the zeta statistic is in fact a structural change statistic test for the parameters of the auxiliary model. Under the alternative (3.3), a Taylor expansion of the score evaluated at $\theta_{t, T}$ yields:

$$
s_{t}\left(\theta_{t, T}\right)=s_{t}\left(\theta^{*}\right)+\frac{\partial s(\tilde{\theta})}{\partial \theta} \frac{h(\pi)}{\sqrt{T}}+o_{p}(1)
$$

and therefore

$$
E s_{t}\left(\theta^{*}\right)=-E \frac{\partial s(\tilde{\theta})}{\partial \theta} \frac{h(\pi)}{\sqrt{T}}+o_{p}(1)
$$

where $\tilde{\theta}$ is defined in the Appendix. Using the result in (4.9) and previous results, we can show that the asymptotic distribution of the zeta statistic under the alternative is given by:

$$
12\left[\int_{0}^{1} B B_{q}(\pi)+I_{T}^{-1 / 2} J(H(\pi)-\pi H(1))\right]^{\prime}\left[\int_{0}^{1} B B_{q}(\pi)+I_{T}^{-1 / 2} J(H(\pi)-\pi H(1))\right]
$$

where $B B(\pi)$ is a vector of independent Browinian Bridge of dimension $q$. The second term in the bracket shows that the zeta statistic is powerful against structural change alternative for the parameter vector $\theta$. However, this test is not an optimal test of structural change as defined by Andrews and Ploberger (1994) and Sowell (1996a,b).

\subsection{Simulated Score Tests}

We introduce a specific structural change test for the Efficient Method of Moments called simulated score tests. The tests rely on simulated series from a restricted null model of interest. Using the reprojection arguments of Gallant and Tauchen (1998), we can fit a sieve seminonparametric SNP density to the simulated data. Under the null, the simulated data 
should yield a reprojection score generator which is a martingale difference sequence when applied to the actual sample data.

In the case of structural change tests, the simulated score test consists of evaluating the score for the actual sample data for a possible breakpoint using the estimator of the auxiliary model for the simulated data. The first step is to simulate series with the restricted estimator defined in equation (2.18). The second step is to obtain the estimator of $\theta$ of the auxiliary model with the simulated series. The score for this second step is:

$$
s\left(\hat{\rho}_{T}^{S}, \hat{\theta}_{N}\right)=\frac{1}{N} \sum_{t=1}^{N} \frac{\partial}{\partial \theta} \log f\left(y_{t}^{s}\left(\hat{\rho}_{T}^{S}\right) \mid Z_{t-1}^{s}\left(\hat{\rho}_{T}^{S}\right), \hat{\theta}_{N}\right) .
$$

where $N$ is the length of the simulated series. The third step is to evaluate the score with the data for a possible breakpoint at the estimator obtained in the second step. The simulated score structural change test is then based on the following statistic:

$$
m_{1}\left(\hat{\theta}_{N}\left(\hat{\rho}_{T}^{S}\right), \pi\right)=\frac{1}{T \pi} \sum_{t=1}^{T \pi} \frac{\partial}{\partial \theta} \log f\left(y_{t} \mid Z_{t-1}, \hat{\theta}_{N}\left(\hat{\rho}_{T}^{S}\right)\right) .
$$

where $\hat{\theta}_{T}\left(\hat{\rho}_{T}^{S}\right)$ is the estimator of the auxiliary model obtained with simulated series. The next theorem gives the asymptotic distribution of the statistic defined above.

Theorem 4.1 Under Assumptions A.2 and D.1 and the alternative (3.3)

$$
\begin{aligned}
\pi \sqrt{T} I_{T}^{-1 / 2} m_{1}\left(\hat{\theta}_{N}\left(\hat{\rho}_{T}^{S}\right), \pi\right) \Rightarrow & B(\pi)+I_{1}^{-1 / 2} J H(\pi)-\pi \frac{\sqrt{T}}{\sqrt{N}} B^{N}(1) \\
& -\pi I^{-1 / 2} M_{\rho}\left[M_{\rho}^{\prime} I^{-1} M_{\rho}\right]^{-1} M_{\rho}^{\prime} I^{-1 / 2} \\
& {\left[B(1)-\frac{1}{\sqrt{S}} B^{s}(1)+I^{-1 / 2} J H(1)\right] }
\end{aligned}
$$

where $H(\pi)=\int_{0}^{\pi} h(\eta, s, u) d u$ and $B(\pi)$ and $B^{N}(\pi)$ are two q-dimensional vectors of mutually independent Brownian motions.

Proof: See Appendix E

Under the null, the asymtotic distribution differs from the one obtained in Theorem 3.3. In particular, it depends on the length of the simulated series $N$. However, replacing $N$ by $T S \pi$ for $\pi \in \Pi$ as the length of the simulated series results in the asymptotic distribution appearing in Theorem 3.3. This is the same argument as the one developed in the discussion of the van der Sluis statistic. Using $T S \pi$ as the length for the simulated series yields asymptotic distributions under the null and under the local alternative that are identical to the distributions of the test proposes in Section 3.2. Structural change tests can then 
be constructed by replacing $I_{T}$ by $\tilde{I}_{T}=\left(1+\frac{1}{S}\right) I_{T}$ as shown in the previous sections. The asymptotic distributions of these structural change tests are given in Proposition 3.1 for the parameter stability and in Proposition 3.2 for the stability of overidentifying restrictions. Hence, the simulated score tests have the same asymptotic distribution under the null and the alternative as the the tests appearing in Section 3.2. However, the small sample properties can differ. In particular, the usual statistics proposed in Section 3.2 are based on the unrestricted estimators $\theta_{i T}$ for $i=1,2$. For small or large value of $\pi \in \Pi$, the properties of the unrestricted estimators $\theta_{i T}$ could be poor since the partial samples used to obtain these estimators are relatively small. This problem does not occur for the computation of the simulated score test.

\section{Conclusions}

Estimation procedures involving auxiliary models are more commonly used, particularly in situations where likelihood-based estimation is infeasible. Many empirical examples can be found in the financial econometrics literature, particularly pertaining to the estimation of continuous time processes. Financial markets experience regular disruptions, sometimes modeled as so called jumps. There may be more fundamental shifts at work and the tests proposed here would be applicable.

Besides generalizing existing test procedures we also introduced new ones which rely on the dual model setup. The simulated score tests introduced in the paper can easily be extended to hypotheses other than structural breaks. As a by product of the paper, we also showed that some recently proposed diagnostic tests for auxiliary models are de facto tests for structural change, albeit suboptimal ones. 


\section{Appendices}

\section{A Technical Assumptions}

To simplify the notation, $\psi\left(y_{t} \mid Z_{t-1}, x_{t} ; \theta\right)$ will be noted $\psi(\theta)$.

\section{A.1 Assumptions for the auxiliary model}

Assumption A.1 $\Psi_{T}(\theta, \pi)$ does not depend on $\pi$ for all $\theta$ under the null hypothesis.

Assumption A.2 The following are assumed to hold:

- $\hat{\rho}_{T}-\rho^{0} \stackrel{p}{\rightarrow} 0$ under the null for some $\rho^{0}$ in the interior of $\Re$.

- $\sup _{\pi \in \Pi}\left\|\hat{\rho}_{i T}(\pi)-\rho^{0}\right\| \stackrel{p}{\rightarrow} 0$ under the null for some $\rho^{0}$ in the interior of $\Re$ for $i=1,2$.

- $\hat{\theta}_{T}-\theta^{*} \stackrel{p}{\rightarrow} 0$ under the null for some $\theta^{*}$ in the interior of $\Theta$.

- $\sup _{\pi \in \Pi}\left\|\hat{\theta}_{i T}(\pi)-\theta^{*}\right\| \stackrel{p}{\rightarrow} 0$ under the null for some $\theta^{*}$ in the interior of $\Theta$ for $i=1,2$.

- $\Psi_{i T}(\theta, \pi)$ is twice continuously partially differential in $\theta$ for all $\theta \in \Theta^{*}$ and $\pi \in \Pi$ with probability one, where $\Theta^{*}$ is some neighborhood of $\theta^{*}$.

- $\frac{\partial^{2} \Psi_{i T}}{\partial \theta \partial \theta^{\prime}}(\theta, \pi) \stackrel{p}{\rightarrow} J$ for $i=1,2$ uniformly over $\pi \in \Pi$ and $\theta \in \Theta^{*}$ where $J=\lim _{T \rightarrow \infty}\left[\frac{1}{T} \sum_{t=1}^{T} \frac{\partial^{2} \psi_{t}}{\partial \theta \partial \theta^{\prime}}\left(\theta^{*}\right)\right]$.

- $I_{i T}(\theta, \pi) \stackrel{p}{\rightarrow} I$ for $i=1,2$ uniformly over $\pi \in \Pi$ and $\theta \in \Theta^{*}$ where $I_{1 T}(\theta, \pi)=$ $\operatorname{var}\left(\frac{1}{\sqrt{[T \pi]}} \sum_{t=1}^{[T \pi]} \frac{\partial \psi_{t}}{\partial \theta}(\theta)\right), I_{2 T}(\theta, \pi)=\operatorname{var}\left(\frac{1}{\sqrt{T-[T \pi]}} \sum_{[T \pi]+1}^{T} \frac{\partial \psi_{t}}{\partial \theta}(\theta)\right)$ and $I=\lim _{T \rightarrow \infty} \operatorname{var}\left(\frac{1}{\sqrt{T}} \sum_{t=1}^{T} \frac{\partial \psi_{t}}{\partial \theta}\left(\theta^{*}\right)\right)$.

- $J$ is uniformly positive definite over $\pi \in \Pi$.

- $\frac{1}{\sqrt{T}} \sum_{t=1}^{[T \pi]} \frac{\partial \psi_{t}}{\partial \theta}\left(\theta^{*}, \pi\right) \Rightarrow I^{1 / 2} B(\pi)-J H(\pi)$ under the alternative (3.3) where $B(\pi)$ is a q-dimensional vector of standard Brownian motions and $H(\pi)=\int_{0}^{\pi} h(r) d r$. 


\section{B Asymptotic Least Squares Regularity Conditions}

Assumption B.1 The following are assumed to hold:

- $g\left(\theta_{i T}(\pi), \rho\right)$ is continuously partially differential in $\theta$ for all $\theta \in \Theta^{*}$ and $\pi \in \Pi$ with probability one, where $\Theta^{*}$ is some neighborhood of $\theta^{*}$ for $i=1,2$.

- $g\left(\theta_{i T}(\pi), \rho\right)$ is continuously partially differential in $\rho$ for all $\rho \in \Re^{0}$ and $\pi \in \Pi$ with probability one, where $\Re^{0}$ is some neighborhood of $\rho^{0}$ for $i=1,2$.

- $\frac{\partial g}{\partial \theta^{\prime}}\left(\hat{\theta}_{i T}(\pi), \rho\right) \stackrel{p}{\rightarrow} G_{\theta}$ for $i=1,2$ uniformly over $\pi \in \Pi$ and $\theta \in \Theta^{*}$ where $G_{\theta}=$ $\frac{\partial g}{\partial \theta^{\prime}}\left(\theta^{*}, \rho^{0}\right)$

- $\frac{\partial g}{\partial \rho}\left(\hat{\theta}_{i T}(\pi), \rho\right) \stackrel{p}{\rightarrow} G_{\rho}$ for $i=1,2$ uniformly over $\pi \in \Pi$ and $\rho \in \Re$ where $G_{\rho}=\frac{\partial g}{\partial \rho}\left(\theta^{*}, \rho^{0}\right)$

- $\sup _{\pi \in \Pi}\left\|W_{i T}(\pi)-W_{0}\right\| \stackrel{p}{\rightarrow} 0$ for $i=1,2$.

\section{Indirect Inference Regularity Conditions}

Assumption C.1 The following are assumed to hold:

- $\hat{\rho}_{T}^{S}-\rho^{0} \stackrel{p}{\rightarrow} 0$ for some $\rho_{0}$ in the interior of $\Re$.

- $\sup _{\pi \in \Pi}\left\|\hat{\rho}_{i T}^{S}(\pi)-\rho^{0}\right\| \stackrel{p}{\rightarrow} 0$ for some $\rho^{0}$ in the interior of $\Re$ for $i=1,2$.

- $\hat{\theta}_{T}^{s}(\rho)-b(\rho) \stackrel{p}{\rightarrow} 0$ for some $\theta^{*}$ in the interior of $\Theta$ for $s=1, \ldots, S$.

- $\sup _{\pi \in \Pi}\left\|\hat{\theta}_{i T}^{s}(\rho, \pi)-b_{i}(\rho, \pi)\right\| \stackrel{p}{\rightarrow} 0$ under the null for some nonrandom function $b_{i}(\rho, \pi)$, for $\rho \in \Re^{0}$ where $\Re^{0}$ is some neighborhood of $\rho^{0}$, for $s=1, \ldots, S$ and $i=1,2$.

- $\Psi_{T}^{s}(\theta, \pi)$ is twice continuously partially differential in $\theta$ for all $\theta \in \Theta^{*}$ and $\pi \in \Pi$ with probability one, where $\Theta^{*}$ is some neighborhood of $\theta^{*}$ and for $s=1, \ldots, S$.

- $\frac{\partial^{2} \Psi_{i}^{s}}{\partial \theta \partial \theta^{\prime}}(\theta, \pi) \stackrel{p}{\rightarrow} J$ for $i=1,2$ uniformly over $\pi \in \Pi$ and $\theta \in \Theta^{*}$ for $s=1, \ldots, S$.

- $I_{i T}^{s}(\theta, \pi) \stackrel{p}{\rightarrow} I$ for $i=1,2$ uniformly over $\pi \in \Pi$ and $\theta \in \Theta^{*}$ where $I_{1 T}^{s}(\theta, \pi)=$ $\operatorname{var}\left(\frac{1}{\sqrt{[T \pi]}} \sum_{t=1}^{[T \pi]} \frac{\partial \psi_{t}^{s}}{\partial \theta}(\theta)\right), I_{2 T}^{s}(\theta, \pi)=\operatorname{var}\left(\frac{1}{\sqrt{T-[T \pi]}} \sum_{[T \pi]+1}^{T} \frac{\partial \psi_{t}^{s}}{\partial \theta}(\theta)\right)$ and for $s=1, \ldots, S$. 
- $\theta_{i T}^{s}(\rho, \pi)$ is continuously partially differentiable in $\rho \in \Re^{0}$ and $\pi \in \Pi$ with probability one for $i=1,2$ and $s=1, \ldots, S$.

- $b(\rho, \pi)$ is continuously partially differentiable in $\rho \in \Re^{0}$ and $\pi \in \Pi$ and is noted $b_{\rho}(\rho, \pi)$.

- $\sup _{\pi \in \Pi}\left\|\frac{\partial \hat{\theta}_{i T}^{s}}{\partial \rho^{\prime}}(\rho, \pi)-b_{\rho}(\rho, \pi)\right\| \stackrel{p}{\rightarrow} 0$ for $i=1,2$ and $s=1, \ldots, S$.

- $\frac{1}{\sqrt{T}} \sum_{t=1}^{[T \pi]} \frac{\partial \psi_{t}^{s}}{\partial \theta}\left(b\left(\rho^{0}\right)\right) \Rightarrow I^{1 / 2} B(\pi)^{s}$ where $B(\pi)^{s}$ is a $q$-dimensional vector of Brownian motions for $s=1, \ldots, S$ and $b\left(\rho^{0}\right)=\theta^{*}$

- $\sup _{\pi \in \Pi}\left\|W_{i T}(\pi)-W_{0}\right\| \stackrel{p}{\rightarrow} 0$ for $i=1,2$.

\section{Efficient Method of Moments Regularity Conditions}

Assumption D.1 The following are assumed to hold:

- $m^{S}\left(\rho, \theta_{i T}(\pi)\right)$ is continuously partially differential in $\theta$ for all $\theta \in \Theta^{*}$ and $\pi \in \Pi$ with probability one, where $\Theta^{*}$ is some neighborhood of $\theta^{*}$ for $i=1,2$.

- $m^{S}\left(\rho, \theta_{i T}(\pi)\right)$ is continuously partially differential in $\rho$ for all $\rho \in \Re^{0}$ and $\pi \in \Pi$ with probability one, where $\Re^{0}$ is some neighborhood of $\rho^{0}$ for $i=1,2$.

- $-\frac{\partial m^{s}}{\partial \theta^{\prime}}\left(\rho, \hat{\theta}_{i T}(\pi)\right) \stackrel{p}{\rightarrow} J$ for $i=1,2$ uniformly over $\pi \in \Pi$ and $\theta \in \Theta^{*}$.

- $\frac{\partial m^{S}}{\partial \rho^{\prime}}\left(\hat{\theta}_{i T}(\pi), \rho\right) \stackrel{p}{\rightarrow} M_{\rho}$ for $i=1,2$ uniformly over $\pi \in \Pi$ and $\rho \in \Re$ where $M_{\rho}=$ $\frac{\partial m^{S}}{\partial \rho}\left(\theta^{*}, \rho^{0}\right)$ 


\section{E Proof of Theorems}

\section{E.1 Proof of Theorem 3.1}

We need to use the following Lemma to proof the Theorem.

Lemma E.1 Under assumptions A.1, A.2, B.1 and the alternative hypothesis (3.3), the asymptotic distribution of the full sample ALS estimator is

$$
\sqrt{T}\left(\hat{\rho}_{T}-\rho_{0}\right) \Rightarrow\left[G_{\rho}^{\prime} W_{0} G_{\rho}\right]^{-1} G_{\rho}^{\prime} W_{0} G_{\theta} J^{-1} I^{1 / 2}\left[B(1)-I^{-1 / 2} J H(1)\right]
$$

and the asymptotic distributions of the unrestricted M-estimators are:

$$
\sqrt{T}\left(\hat{\theta}_{1 T}(\pi)-\theta^{*}\right) \Rightarrow-J^{-1} I^{1 / 2}\left[\frac{B(\pi)-I^{1 / 2} J H(\pi)}{\pi}\right]
$$

and

$$
\sqrt{T}\left(\hat{\theta}_{2 T}(\pi)-\theta^{*}\right) \Rightarrow-J^{-1} I^{1 / 2}\left[\frac{B(1)-B(\pi)-I^{1 / 2} J(H(1)-H(\pi))}{(1-\pi)}\right] .
$$

\section{Proof of Lemma E.1:}

First, the asymptotic distribution for the restricted estimator is shown. By the mean value expansion for the F.O.C. evaluated at $\theta_{T}$ :

$$
\frac{1}{T} \sum_{t=1}^{T} \frac{\partial \psi_{t}\left(\hat{\theta}_{T}\right)}{\partial \theta}=\frac{1}{T} \sum_{t=1}^{T} \frac{\partial \psi_{t}\left(\theta^{*}\right)}{\partial \theta}+\frac{1}{T} \sum_{t=1}^{T} \frac{\partial^{2} \psi_{t}(\tilde{\theta})}{\partial \theta \partial \theta^{\prime}}\left(\hat{\theta}_{T}-\theta^{*}\right)+o_{p}(1)
$$

where $\tilde{\theta}^{\prime}=\left[\tilde{\theta}^{(1)} \ldots \tilde{\theta}^{(q)}\right]$ and $\tilde{\theta}^{(k)}=\lambda^{(k)} \theta_{T}^{*(k)}+\left(1-\lambda^{(k)}\right) \hat{\theta}^{(k)}$ for some $0 \leq \lambda^{(k)} \leq 1$ and $k=1, \ldots, q$. The expression above yields:

$$
\sqrt{T}\left(\hat{\theta}_{T}-\theta^{*}\right)=-\left[\frac{1}{T} \sum_{t=1}^{T} \frac{\partial^{2} \psi_{t}(\tilde{\theta})}{\partial \theta \partial \theta^{\prime}}\right]^{-1} \frac{1}{\sqrt{T}} \sum_{t=1}^{T} \frac{\partial \psi_{t}\left(\theta^{*}\right)}{\partial \theta}+o_{p}(1) .
$$

By Assumption A.2, we have

$$
\frac{1}{\sqrt{T}} \sum_{t=1}^{T} \frac{\partial \psi_{t}\left(\theta^{*}\right)}{\partial \theta} \stackrel{p}{\rightarrow} I^{1 / 2} B(1)-J H(1)
$$

where $H(1)=\int_{0}^{1} h(r) d r$. Since $\hat{\theta}_{T}$ is consistent for $\theta^{*}, \tilde{\theta} \stackrel{p}{\rightarrow} \theta^{*}$ and by Assumption A.2, the asymptotic distribution of the full sample estimator $\hat{\theta}_{T}$ is then given by

$$
\sqrt{T}\left(\hat{\theta}_{T}-\theta^{*}\right) \stackrel{d}{\rightarrow}-J^{-1} I^{1 / 2}\left[B(1)-I^{-1 / 2} J H(1)\right]
$$


The mean value expansion for the restricted ALS estimator is

$$
\begin{aligned}
\left(\frac{\partial g\left(\hat{\theta}_{T}, \hat{\rho}_{T}\right)}{\partial \rho^{\prime}}\right)^{\prime} W_{T} g\left(\hat{\theta}_{T}, \hat{\rho}_{T}\right)= & \left(\frac{\partial g\left(\hat{\theta}_{T}, \hat{\rho}_{T}\right)}{\partial \rho^{\prime}}\right)^{\prime} W_{T} g\left(\theta^{*}, \rho^{0}\right) \\
& +\left(\frac{\partial g\left(\hat{\theta}_{T}, \hat{\rho}_{T}\right)}{\partial \rho^{\prime}}\right)^{\prime} W_{T} \frac{\partial g}{\partial \theta^{\prime}}\left(\tilde{\theta}, \hat{\rho}_{T}\right)\left(\hat{\theta}_{T}-\theta^{*}\right) \\
& +\left(\frac{\partial g\left(\hat{\theta}_{T}, \hat{\rho}_{T}\right)}{\partial \rho^{\prime}}\right)^{\prime} W_{T} \frac{\partial g}{\partial \rho^{\prime}}(\hat{\theta}, \tilde{\rho})\left(\hat{\rho}_{T}-\rho^{0}\right)+o_{p}(1)
\end{aligned}
$$

where $\tilde{\rho}^{\prime}=\left[\tilde{\rho}^{(1)} \ldots \tilde{\rho}^{(p)}\right]$ and $\tilde{\rho}^{(k)}=\lambda^{(k)} \rho^{0,(k)}+\left(1-\lambda^{(k)}\right) \hat{\rho}^{(k)}$ for some $0 \leq \lambda^{(k)} \leq 1$ and $k=1, \ldots, p$ and $\tilde{\theta}$ is defined above.

By the fact that the first term of the right hand side is equal to zero, the full sample estimator $\hat{\rho}_{T}$ is only function of the asymptotic distribution of $\hat{\theta}_{T}$. Thus, by the expansion above, we have

$$
\begin{aligned}
\sqrt{T}\left(\hat{\rho}_{T}-\rho^{0}\right)= & -\left[\left(\frac{\partial g\left(\hat{\theta}_{T}, \hat{\rho}_{T}\right)}{\partial \rho^{\prime}}\right)^{\prime} W_{T} \frac{\partial g}{\partial \rho^{\prime}}(\hat{\theta}, \tilde{\rho})\right]^{-1} \\
& \times\left(\frac{\partial g\left(\hat{\theta}_{T}, \hat{\rho}_{T}\right)}{\partial \rho^{\prime}}\right)^{\prime} W_{T} \frac{\partial g}{\partial \theta^{\prime}}\left(\tilde{\theta}_{T}, \hat{\rho}_{T}\right) \sqrt{T}\left(\hat{\theta}_{T}-\theta^{*}\right)+o_{p}(1) .
\end{aligned}
$$

Since $\hat{\theta}_{T}$ is consistent for $\theta^{*}$, then $\tilde{\theta} \stackrel{p}{\rightarrow} \theta^{*}$ and $\hat{\rho}_{T}$ is consistent for $\rho^{0}$ then $\tilde{\rho} \stackrel{p}{\rightarrow} \rho^{0}$. Under Assumptions B.1, the consistency of $\tilde{\theta}$ and $\tilde{\rho}$ and the result (E.2), we obtain that

$$
\sqrt{T}\left(\hat{\rho}_{T}-\rho^{0}\right) \stackrel{p}{\rightarrow}\left[G_{\rho}^{\prime} W_{0} G_{\rho}\right]^{-1} G_{\rho}^{\prime} W_{0} G_{\theta} J^{-1} I^{1 / 2}\left[B(1)-I^{-1 / 2} J H(1)\right] .
$$

The optimal estimator is obtained with the following weighting matrix

$$
W_{T}=\Omega_{T}^{-1}=\left[\frac{\partial g}{\partial \theta^{\prime}} J_{T}^{-1} I_{T} J_{T}^{-1}\left(\frac{\partial g}{\partial \theta^{\prime}}\right)^{\prime}\right]^{-1}
$$

and

$$
\Omega_{T} \stackrel{p}{\rightarrow} \Omega=\left[G_{\theta} J^{-1} I J^{-1} G_{\theta}^{\prime}\right] .
$$

Now, we derive the asymptotic distribution for the unrestricted estimators. By the mean value expansion for the M-estimators $\hat{\theta}_{1 T}$ for the first subsamples:

$$
\frac{1}{[T \pi]} \sum_{t=1}^{[T \pi]} \frac{\partial \psi_{t}\left(\hat{\theta}_{1 T}\right)}{\partial \theta}=\frac{1}{[T \pi]} \sum_{t=1}^{[T \pi]} \frac{\partial \psi_{t}\left(\theta^{*}\right)}{\partial \theta}+\frac{1}{[T \pi]} \sum_{t=1}^{[T \pi]} \frac{\partial^{2} \psi_{t}\left(\tilde{\theta}_{1}\right)}{\partial \theta \partial \theta^{\prime}}\left(\hat{\theta}_{1 T}(\pi)-\theta^{*}\right)+o_{p}(1)
$$

where $\tilde{\theta}_{1}{ }^{\prime}=\left[\tilde{\theta}_{1}^{(1)} \ldots \tilde{\theta}_{1}^{(q)}\right]$ and $\tilde{\theta}_{1}^{(k)}=\lambda^{(k)} \theta_{1}^{*(k)}+\left(1-\lambda^{(k)}\right) \hat{\theta}_{1 T}^{(k)}$ for some $0 \leq \lambda^{(k)} \leq 1$ and $k=1, \ldots, q$. Since $\hat{\theta}_{1 T}$ is consistent for $\theta^{*}$ by Assumption A. $2, \bar{\theta} \stackrel{p}{\rightarrow} \theta^{*}$. This yields

$$
\sqrt{T}\left(\hat{\theta}_{1 T}(\pi)-\theta^{*}\right)=-\left(\frac{1}{[T \pi]} \sum_{t=1}^{[T \pi]} \frac{\partial^{2} \psi_{t}\left(\tilde{\theta}_{1}\right)}{\partial \theta \partial \theta^{\prime}}\right)^{-1} \sqrt{T} \frac{1}{[T \pi]} \sum_{t=1}^{[T \pi]} \frac{\partial \psi_{t}\left(\theta^{*}\right)}{\partial \theta}+o_{p}(1) .
$$


Similarly, by the mean value expansion of the M-estimators for the second subsamples and manipulating this expansion, we obtain

$$
\begin{aligned}
\sqrt{T}\left(\hat{\theta}_{2 T}(\pi)-\theta^{*}\right)= & -\left(\frac{1}{[T(1-\pi)]} \sum_{t=[T \pi]+1}^{T} \frac{\partial^{2} \psi_{t}\left(\tilde{\theta}_{2}\right)}{\partial \theta \partial \theta^{\prime}}\right)^{-1} \\
& \sqrt{T} \frac{1}{[T(1-\pi)]} \sum_{t=[T \pi]+1}^{T} \frac{\partial \psi_{t}\left(\theta^{*}\right)}{\partial \theta}+o_{p}(1) .
\end{aligned}
$$

Using the weak convergence of the score (assumption A.2), we have

$$
\sqrt{T} \frac{1}{[T \pi]} \sum_{t=1}^{[T \pi]} \frac{\partial \psi_{1 t}\left(\theta^{*}\right)}{\partial \theta} \Rightarrow I^{1 / 2}\left[\frac{B(\pi)-I^{-1 / 2} J H(\pi)}{\pi}\right]
$$

and

$$
\sqrt{T} \frac{1}{[T(1-\pi)]} \sum_{t=[T \pi]+1}^{T} \frac{\partial \psi_{2 t}\left(\theta^{*}\right)}{\partial \theta} \Rightarrow I^{1 / 2}\left[\frac{B(1)-B(\pi)-I^{-1 / 2} J(H(1)-H(\pi))}{(1-\pi)}\right] .
$$

Under Assumption A.2, the asymptotic distribution of the unrestricted M-estimators are then:

$$
\begin{gathered}
\sqrt{T}\left(\hat{\theta}_{1 T}(\pi)-\theta^{*}\right) \Rightarrow-J^{-1} I^{1 / 2}\left[\frac{B(\pi)-I^{-1 / 2} J H(\pi)}{\pi}\right] \\
\sqrt{T}\left(\hat{\theta}_{2 T}(\pi)-\theta^{*}\right) \Rightarrow-J^{-1} I^{1 / 2}\left[\frac{B(1)-B(\pi)-I^{-1 / 2} J(H(1)-H(\pi))}{(1-\pi)}\right] .
\end{gathered}
$$

\section{Proof of Theorem 3.1:}

First, we show the result for the first subsample. We do the mean value expansion for $\Omega_{T}^{-1 / 2} g\left(\hat{\theta}_{1 T}(\pi), \hat{\rho}_{T}\right)$ which yields

$$
\begin{aligned}
\Omega_{T}^{-1 / 2} g\left(\hat{\theta}_{1 T}(\pi), \hat{\rho}_{T}\right)= & \Omega_{T}^{-1 / 2} g\left(\theta^{*}, \rho^{0}\right)+\Omega_{T}^{-1 / 2} \frac{\partial g}{\partial \theta^{\prime}}\left(\tilde{\theta}_{1}(\pi), \hat{\rho}_{T}\right)\left(\hat{\theta}_{1 T}-\theta^{*}\right) \\
& +\Omega_{T}^{-1 / 2} \frac{\partial g}{\partial \rho^{\prime}}\left(\hat{\theta}_{1 T}(\pi), \tilde{\rho}\right)\left(\hat{\rho}_{T}-\rho^{0}\right)+o_{p}(1)
\end{aligned}
$$

where $\tilde{\theta}_{1}(\pi)$ and $\tilde{\rho}$ are defined above. By using Lemma E.1, Assumptions A.2 and B.1 with the convergence in probability of $\Omega_{T}$ to $\Omega$, we obtain that

$$
\begin{aligned}
\sqrt{T} \Omega_{T}^{-1 / 2} g\left(\hat{\theta}_{1 T}(\pi), \hat{\rho}_{T}\right) \Rightarrow & -\Omega^{-1 / 2} G_{\theta} J^{-1} I^{1 / 2}\left[\frac{B(\pi)-I^{-1 / 2} J H(\pi)}{\pi}\right] \\
& +\Omega^{-1 / 2} G_{\rho}\left(G_{\rho}^{\prime} S^{-1} G_{\rho}\right)^{-1} G_{\rho} \Omega^{-1} G_{\theta} J I^{1 / 2}\left[B(1)-I^{-1 / 2} J H(1)\right] .
\end{aligned}
$$

Since $\Omega^{-1 / 2} G_{\theta} J^{-1} I^{1 / 2} B(\pi)$ is a q-dimensional standard Brownian motions, the result follows. The asymptotic distribution for the second sample can be obtained in a similar way. 


\section{E.2 Proof of Theorem 3.2}

Lemma E.2 Under assumptions A.2, C.1 and the alternative (3.3), the asymptotic distribution of the indirect inference estimator is

$$
\sqrt{T}\left(\hat{\rho}_{T}^{S}-\rho_{0}\right) \stackrel{p}{\rightarrow}\left[b_{\rho}^{\prime} W_{0} b_{\rho}\right]^{-1} b_{\rho}^{\prime} W_{0} J I^{1 / 2}\left[B(1)-\frac{1}{S} \sum_{s=1}^{S} B^{s}(1)-I^{-1 / 2} J H(1)\right]
$$

and the asymptotic distributions of the unrestricted simulated M-estimators are:

$$
\begin{gathered}
\sqrt{T}\left(\hat{\theta}_{1 T}^{s}\left(\rho^{0}, \pi\right)-\theta^{*}\right) \Rightarrow-J^{-1} I^{1 / 2} \frac{B(\pi)}{\pi} \\
\sqrt{T}\left(\hat{\theta}_{2 T}^{s}\left(\rho^{0}, \pi\right)-\theta^{*}\right) \Rightarrow-J^{-1} I^{1 / 2}\left[\frac{B(1)-B(\pi)}{(1-\pi)}\right]
\end{gathered}
$$

\section{Proof of Lemma E.2:}

Now, we derive the asymptotic distribution of the restricted estimator $\hat{\rho}^{S}$. For the simulated path $s$, we have the following mean value expansion for the F.O.C. evaluated at $\hat{\theta}^{s}$ :

$$
\frac{1}{T} \sum_{t=1}^{T} \frac{\partial \psi_{t}^{s}\left(\hat{\theta}_{T}^{s}\right)}{\partial \theta}=\frac{1}{T} \sum_{t=1}^{T} \frac{\partial \psi_{t}^{s}\left(\theta^{*}\right)}{\partial \theta}+\frac{1}{T} \sum_{t=1}^{T} \frac{\partial^{2} \psi_{t}^{s}\left(\tilde{\theta}^{s}\right)}{\partial \theta \partial \theta^{\prime}}\left(\hat{\theta}_{T}^{s}-\theta^{*}\right)+o_{p}(1)
$$

where $\tilde{\theta}^{s}$ is defined as $\tilde{\theta}$ in the proof of Lemma E.1 for a simulated path $s$. Then

$$
\sqrt{T}\left(\hat{\theta}_{T}^{s}-\theta^{*}\right)=-\left[\frac{1}{T} \sum_{t=1}^{T} \frac{\partial^{2} \psi_{t}^{s}\left(\tilde{\theta}^{s}\right)}{\partial \theta \partial \theta^{\prime}}\right]^{-1} \sqrt{T} \frac{1}{T} \sum_{t=1}^{T} \frac{\partial \psi_{t}^{s}\left(\theta^{*}\right)}{\partial \theta}+o_{p}(1) .
$$

Under Assumption C.1 and by the consistency of $\tilde{\theta}^{s}$, the asymptotic distribution of $\hat{\theta}^{s}$ is given by:

$$
\sqrt{T}\left(\hat{\theta}_{T}^{s}-\theta^{*}\right) \stackrel{d}{\rightarrow}-J^{-1} I^{1 / 2} B(1)^{s}
$$

In contrast to the asymptotic distribution of the estimator $\theta\left(\hat{\theta}_{T}\right)$ obtained for with data, the asymptotic distribution of $\hat{\theta}_{T}^{s}$ does not depend on the alternative for obvious reasons. The mean value expansion for the restricted indirect inference estimator is 


$$
\begin{aligned}
{\left[\frac{1}{S} \sum_{s=1}^{S} \frac{\partial \hat{\theta}_{T}^{s}}{\partial \rho^{\prime}}\left(\hat{\rho}_{T}^{S}\right)\right]^{\prime} W_{T}\left[\hat{\theta}_{T}-\frac{1}{S} \sum_{s=1}^{S} \theta_{T}^{s}\left(\hat{\rho}_{T}^{S}\right)\right]=} & {\left[\frac{1}{S} \sum_{s=1}^{S} \frac{\partial \hat{\theta}_{T}^{s}}{\partial \rho^{\prime}}\left(\hat{\rho}_{T}^{S}\right)\right]^{\prime} W_{T} } \\
& {\left[\left(\hat{\theta}_{T}-\theta^{*}\right)-\frac{1}{S} \sum_{s=1}^{S}\left(\hat{\theta}_{T}^{s}\left(\hat{\rho}_{T}^{S}\right)-\theta^{*}\right)\right.} \\
& \left.-\left(\frac{1}{S} \sum_{s=1}^{S} \frac{\partial \hat{\theta}_{T}^{s}}{\partial \rho^{\prime}}\left(\tilde{\rho}_{T}^{S}\right)\right)\left(\hat{\rho}_{T}^{S}-\rho_{0}\right)\right]+o_{p}(1)
\end{aligned}
$$

where $\tilde{\rho}^{S}$ is defined as in the proof of Lemma E.1 for the estimator obtained with $S$ simulated paths. This yields:

$$
\begin{gathered}
\sqrt{T}\left(\hat{\rho}_{T}^{S}-\rho_{0}\right)=-\left[\left[\frac{1}{S} \sum_{s=1}^{S} \frac{\partial \theta_{T}^{s}}{\partial \rho^{\prime}}\left(\hat{\rho}_{T}^{S}\right)\right]^{\prime} W_{T} \frac{1}{S} \sum_{s=1}^{S} \frac{\partial \hat{\theta}_{T}^{s}}{\partial \rho^{\prime}}\left(\tilde{\rho}_{T}^{S}\right)\right]^{-1} \\
\times\left[\frac{1}{S} \sum_{s=1}^{S} \frac{\partial \hat{\theta}_{T}^{s}}{\partial \rho^{\prime}}\left(\hat{\rho}_{T}^{S}\right)\right]^{\prime} W_{T} \sqrt{T}\left[\left(\hat{\theta}_{T}-\theta^{*}\right)-\frac{1}{S} \sum_{s=1}^{S}\left(\hat{\theta}_{T}^{s}\left(\hat{\rho}_{T}^{S}\right)-\theta^{*}\right)\right]+o_{p}(1) .
\end{gathered}
$$

By Assumptions C.1, Lemma E.1, result (E.3) and the consistency of $\tilde{\rho}_{T}^{S}$, the asymptotic distribution of $\hat{\rho}_{T}^{S}$ is given by:

$$
\sqrt{T}\left(\hat{\rho}_{T}^{S}-\rho_{0}\right) \stackrel{d}{\rightarrow}\left[b_{\rho}^{\prime} W_{0} b_{\rho}\right]^{-1} b_{\rho}^{\prime} W_{0} J^{-1} I^{1 / 2}\left[B(1)-\frac{1}{S} \sum_{s=1}^{S} B^{s}(1)-I^{-1 / 2} J H(1)\right]
$$

The asymptotic distribution depends on the matrice $W_{0}$ and the number of simulations $S$. The restricted optimal Indirect Inference estimator is obtained with the following weighting matrix

$$
W_{T}=\Omega_{T}^{-1}=\left[J_{T}^{-1} I_{T} J_{T}^{-1}\right]^{-1}
$$

and

$$
\Omega_{T} \stackrel{p}{\rightarrow} \Omega=J^{-1} I J^{-1}
$$

Now, we derive the asymptotic distribution for the unrestricted estimators. The mean value expansion of the M-estimators for the first subsample evaluated at $\hat{\theta}_{1 T}\left(\rho^{0}, \pi\right)$ gives

$$
\frac{1}{[T \pi]} \sum_{t=1}^{[T \pi]} \frac{\partial \psi_{t}^{s}\left(\hat{\theta}_{i T}^{s}\left(\rho^{0}\right)\right)}{\partial \theta}=\frac{1}{[T \pi]} \sum_{t=1}^{[T \pi]} \frac{\partial \psi_{t}^{s}\left(\theta^{*}\right)}{\partial \theta}+\frac{1}{[T \pi]} \sum_{t=1}^{[T \pi]} \frac{\partial^{2} \psi_{t}^{s}\left(\tilde{\theta}^{s}\left(\rho^{0}\right)\right)}{\partial \theta \partial \theta^{\prime}}\left(\hat{\theta}_{1 T}^{s}\left(\rho^{0}, \pi\right)-\theta^{*}\right)+o_{p}(1) .
$$

This yields the following expression:

$$
\sqrt{T}\left(\hat{\theta}_{1 T}^{s}\left(\rho^{0}, \pi\right)-\theta^{*}\right)=-\left(\frac{1}{[T \pi]} \sum_{t=1}^{[T \pi]} \frac{\partial^{2} \psi_{t}^{s}\left(\tilde{\theta}^{s}\left(\rho^{0}\right)\right)}{\partial \theta \partial \theta^{\prime}}\right) \frac{1}{\sqrt{[T \pi]}} \sum_{t=1}^{[T \pi]} \frac{\partial \psi_{t}^{s}\left(\theta^{*}\right)}{\partial \theta}+o_{p}(1) .
$$


We can obtain the equivalent expression for $\hat{\theta}_{2 T}(\pi)$ by a similar mean value expansion.

By Assumption C.1, we have the following weak convergence of the score for the first and the second subsamples:

$$
\sqrt{T} \frac{1}{[T \pi]} \sum_{t=1}^{[T \pi]} \frac{\partial \psi_{1 t}^{s}\left(\theta^{*}\right)}{\partial \theta} \Rightarrow I^{1 / 2} \frac{B(\pi)}{\pi}
$$

and

$$
\sqrt{T} \frac{1}{[T(1-\pi)]} \sum_{t=[T \pi]+1}^{T} \frac{\partial \psi_{2 t}^{s}\left(\theta^{*}\right)}{\partial \theta} \Rightarrow I^{1 / 2} \frac{B(1)-B(\pi)}{(1-\pi)} .
$$

Given the results above, Assumption C.1 ans the consistency of $\tilde{\theta}^{s}\left(\rho^{0}\right)$ for $i=1,2$, the asymptotic distributions of the unrestricted simulated M-estimators are respectively:

$$
\begin{gathered}
\sqrt{T}\left(\hat{\theta}_{1 T}^{s}\left(\rho^{0}, \pi\right)-\theta^{*}\right) \Rightarrow-J^{-1} I^{1 / 2} \frac{B(\pi)^{s}}{\pi} \\
\sqrt{T}\left(\hat{\theta}_{2 T}^{s}\left(\rho^{0}, \pi\right)-\theta^{*}\right) \Rightarrow-J^{-1} I^{1 / 2}\left[\frac{B(1)^{s}-B(\pi)^{s}}{(1-\pi)}\right]
\end{gathered}
$$

\section{Proof of Theorem 3.2:}

By a mean value expansion for the first subsample, we have that:

$$
\begin{aligned}
\Omega_{T}^{-1 / 2}\left[\hat{\theta}_{1 T}(\pi)-\frac{1}{S} \sum_{s=1}^{S} \hat{\theta}_{1 T}^{s}\left(\rho_{T}^{S}, \pi\right)\right]= & \Omega_{T}^{-1 / 2}\left[\left(\hat{\theta}_{1 T}(\pi)-\theta^{*}\right)-\frac{1}{S} \sum_{s=1}^{S}\left(\hat{\theta}_{1 T}^{s}\left(\rho^{0}, \pi\right)-\theta^{*}\right)\right] \\
& \Omega_{T}^{-1 / 2}\left[\left(\frac{1}{S} \sum_{s=1}^{S} \frac{\partial \hat{\theta}_{1 T}^{s}}{\partial \rho^{\prime}}\left(\tilde{\rho}_{T}^{S}, \pi\right)\right)\left(\hat{\rho}_{T}^{S}-\rho_{0}\right)\right]+o_{p}(1)
\end{aligned}
$$

By Lemma E.1 and E.2, Assumption C.1 and the consistency of $\tilde{\rho}_{T}^{S}$, we obtain that:

$$
\begin{array}{r}
\sqrt{T} \Omega_{T}^{-1 / 2}\left[\hat{\theta}_{1 T}^{s}-\frac{1}{S} \sum_{s=1}^{S} \hat{\theta}_{1 T}^{s}\left(\rho_{T}^{s}, \pi\right)\right] \Rightarrow-\Omega^{-1 / 2} J^{-1} I^{1 / 2}\left[\frac{B(\pi)}{\pi}-\frac{1}{S} \sum_{s=1}^{S} \frac{B(\pi)^{s}}{\pi}-\frac{I^{-1 / 2} J H(\pi)}{\pi}\right] \\
+\Omega^{-1 / 2} b_{\rho}\left[b_{\rho}^{\prime} \Omega^{-1} b_{\rho}\right]^{-1} b_{\rho}^{\prime} \Omega^{-1} J^{-1} I^{1 / 2}\left[B(1)-\frac{1}{S} \sum_{s=1}^{S} B(1)^{s}-I^{1 / 2} J H(1)\right]
\end{array}
$$

Since $\Omega^{-1 / 2} J^{-1} I^{1 / 2} B(\pi)$ is a $q$-dimensional vector of standard Brownian motions, the result follows. The asymptotic distribution for the second sample is obtained similarly.

\section{E.3 Proof of Theorems 3.3 and 4.1}

Lemma E.3 Under assumptions A.2, D.1 and the alternative hypothesis (3.3), the asymptotic distribution of the full sample EMM estimator is

$$
\sqrt{T}\left(\hat{\rho}_{T}^{S}-\rho_{0}\right) \Rightarrow\left[M_{\rho}^{\prime} I^{-1} M_{\rho}\right]^{-1} M_{\rho}^{\prime} I^{-1 / 2}\left[B(1)-\frac{1}{\sqrt{S}} B(1)^{S}+I^{-1 / 2} J H(1)\right] .
$$




\section{Proof of Lemma E.3:}

First, the asymptotic distribution for the restricted estimator is shown. By the mean value expansion for the F.O.C. evaluated at $\theta_{T}$ :

$$
\frac{1}{T} \sum_{t=1}^{T} \frac{\partial \log f_{t}\left(\hat{\theta}_{T}\right)}{\partial \theta}=\frac{1}{T} \sum_{t=1}^{T} \frac{\partial \log f_{t}\left(\theta^{*}\right)}{\partial \theta}+\frac{1}{T} \sum_{t=1}^{T} \frac{\partial^{2} \log f_{t}(\tilde{\theta})}{\partial \theta \partial \theta^{\prime}}\left(\hat{\theta}_{T}-\theta^{*}\right)+o_{p}(1)
$$

where $\tilde{\theta}^{\prime}=\left[\tilde{\theta}^{(1)} \ldots \tilde{\theta}^{(q)}\right]$ and $\tilde{\theta}^{(k)}=\lambda^{(k)} \theta_{T}^{*(k)}+\left(1-\lambda^{(k)}\right) \hat{\theta}^{(k)}$ for some $0 \leq \lambda^{(k)} \leq 1$ and $k=1, \ldots, q$. The expression above yields:

$$
\sqrt{T}\left(\hat{\theta}_{T}-\theta^{*}\right)=-\left[\frac{1}{T} \sum_{t=1}^{T} \frac{\partial^{2} \log f_{t}(\tilde{\theta})}{\partial \theta \partial \theta^{\prime}}\right]^{-1} \frac{1}{\sqrt{T}} \sum_{t=1}^{T} \frac{\partial \log f_{t}\left(\theta^{*}\right)}{\partial \theta}+o_{p}(1) .
$$

By Assumption A.2, we have

$$
\frac{1}{\sqrt{T}} \sum_{t=1}^{T} \frac{\partial \log f_{t}\left(\theta^{*}\right)}{\partial \theta} \stackrel{p}{\rightarrow} I^{1 / 2} B(1)+J H(1) .
$$

where $J=\lim _{T \rightarrow \infty}\left[-\frac{1}{T} \sum_{t=1}^{T} \frac{\partial^{2} \log f_{t}}{\partial \theta \partial \theta^{\prime}}\left(\theta^{*}\right)\right]$. Since $\hat{\theta}_{T}$ is consistent for $\theta^{*}, \tilde{\theta} \stackrel{p}{\rightarrow} \theta^{*}$, the asymptotic distribution of the full sample estimator $\hat{\theta}_{T}$ is then given by

$$
\sqrt{T}\left(\hat{\theta}_{T}-\theta^{*}\right) \stackrel{d}{\rightarrow} J^{-1} I^{1 / 2}\left[B(1)+I^{-1 / 2} J H(1)\right]
$$

The mean value expansion of the F.O.C. evaluated at the unrestricted EMM estimator is

$$
\begin{aligned}
\left(\frac{\partial m_{T}^{S}\left(\hat{\rho}_{T}^{S}, \hat{\theta}_{T}\right)}{\partial \rho^{\prime}}\right)^{\prime} I_{T}^{-1} m_{T}^{S}\left(\hat{\rho}_{T}^{S}, \hat{\theta}_{T}\right)= & \left(\frac{\partial m_{T}^{S}\left(\hat{\rho}_{T}^{S}, \hat{\theta}_{T}\right)}{\partial \rho^{\prime}}\right)^{\prime} I_{T}^{-1} m_{T}^{S}\left(\rho^{0}, \theta^{*}\right) \\
& +\left(\frac{\partial m_{T}^{S}\left(\hat{\rho}_{T}^{S}, \hat{\theta}_{T}\right)}{\partial \rho^{\prime}}\right)^{\prime} I_{T}^{-1} \frac{\partial m_{T}^{S}}{\partial \theta^{\prime}}\left(\tilde{\theta}, \hat{\rho}_{T}^{S}\right)\left(\hat{\theta}_{T}-\theta^{*}\right) \\
& +\left(\frac{\left.\partial m_{T}^{S}\left(\hat{\rho}_{T}^{S}, \hat{\theta}_{T}\right)\right)}{\partial \rho^{\prime}}\right)^{\prime} I_{T}^{-1} \frac{\partial m_{T}^{S}}{\partial \rho^{\prime}}(\hat{\theta}, \tilde{\rho})\left(\hat{\rho}_{T}^{S}-\rho^{0}\right)+o_{p}(1)
\end{aligned}
$$

where $\tilde{\rho}^{\prime}=\left[\tilde{\rho}^{(1)} \ldots \tilde{\rho}^{(p)}\right]$ and $\tilde{\rho}^{(k)}=\lambda^{(k)} \rho^{0,(k)}+\left(1-\lambda^{(k)}\right) \hat{\rho}^{S,(k)}$ for some $0 \leq \lambda^{(k)} \leq 1$ and $k=1, \ldots, p$ and $\tilde{\theta}$ is defined as in Lemma E.1.

Thus, by the expansion above, we have

$$
\begin{aligned}
\sqrt{T}\left(\hat{\rho}_{T}^{S}-\rho_{0}\right)= & -\left[\left(\frac{\partial m_{T}^{S}\left(\hat{\rho}_{T}^{S}, \hat{\theta}_{T}\right)}{\partial \rho^{\prime}}\right)^{\prime} I_{T}^{-1} \frac{\partial m_{T}^{S}}{\partial \rho^{\prime}}(\hat{\theta}, \tilde{\rho})\right]^{-1}\left(\frac{\partial m_{T}^{S}\left(\hat{\rho}_{T}^{S}, \hat{\theta}_{T}\right)}{\partial \rho^{\prime}}\right)^{\prime} I_{T}^{-1} \\
& \times\left[m_{T}^{S}\left(\rho^{0}, \theta^{*}\right)+\frac{\partial m_{T}^{S}}{\partial \theta^{\prime}}\left(\tilde{\theta}_{T}, \hat{\rho}_{T}^{S}\right) \sqrt{T}\left(\hat{\theta}_{T}-\theta^{*}\right)\right]+o_{p}(1) .
\end{aligned}
$$


Under Assumptions A.2 and D.1 and the result E.5,we obtain that

$$
\sqrt{T}\left(\hat{\rho}_{T}^{S}-\rho_{0}\right) \Rightarrow\left[M_{\rho}^{\prime} I^{-1} M_{\rho}\right]^{-1} M_{\rho}^{\prime} I^{-1 / 2}\left[B(1)-\frac{1}{\sqrt{S}} B(1)^{S}+I^{-1 / 2} J H(1)\right]
$$

since

$$
\sqrt{T} m_{T}^{S}\left(\rho^{0}, \theta^{*}\right) \Rightarrow \frac{1}{\sqrt{S}} I^{1 / 2} B(1)^{S}
$$

and $\frac{\partial m_{T}^{S}}{\partial \theta^{\prime}}\left(\tilde{\theta}_{T}, \hat{\rho}_{T}^{S}\right)$ converge in probability to $-J$. In the case where $S=\infty$, the term $B(1)^{S}$ disappears since $\frac{1}{\sqrt{S}}=0$.

Moreover, we can show that under the alternative the asymptotic distributions of the unrestricted (quasi) maximum likelihood estimators is given by:

$$
\sqrt{T}\left(\hat{\theta}_{1 T}^{s}\left(\rho^{0}, \pi\right)-\theta^{*}\right) \Rightarrow J^{-1} I^{1 / 2}\left[\frac{B(\pi)^{s}+I^{-1 / 2} J H(\pi)}{\pi}\right]
$$

and

$$
\sqrt{T}\left(\hat{\theta}_{2 T}^{s}\left(\rho^{0}, \pi\right)-\theta^{*}\right) \Rightarrow J^{-1} I^{1 / 2}\left[\frac{B(1)^{s}-B(\pi)^{s}+I^{-1 / 2} J(H(1)-H(\pi))}{(1-\pi)}\right] .
$$

\section{Proof of Theorem 3.3:}

First, we show the result for the first subsample. We do the mean value expansion for $I_{T}^{-1 / 2} m_{1}^{S}\left(\hat{\rho}_{T}, \hat{\theta}_{1 T}(\pi)\right)$ which gives

$$
\begin{aligned}
I_{T}^{-1 / 2} m_{1}^{S}\left(\hat{\rho}_{T}^{S}, \hat{\theta}_{1 T}(\pi)\right)= & I_{T}^{-1 / 2} m_{1}^{S}\left(\theta^{*}, \rho^{0}\right)+I_{T}^{-1 / 2} \frac{\partial m_{1}^{S}}{\partial \theta^{\prime}}\left(\tilde{\theta}_{1}(\pi), \hat{\rho}_{T}^{S}\right)\left(\hat{\theta}_{1 T}(\pi)-\theta^{*}\right) \\
& +I_{T}^{-1 / 2} \frac{\partial m_{1}^{S}}{\partial \rho^{\prime}}\left(\hat{\theta}_{1 T}(\pi), \tilde{\rho}\right)\left(\hat{\rho}_{T}-\rho^{0}\right)+o_{p}(1)
\end{aligned}
$$

By using Lemma E.1 and E.3, Assumptions A.2, D.1, result E.6 and the consistency of $\tilde{\theta}_{1}(\pi)$ and $\tilde{\rho}$, we obtain that

$$
\begin{aligned}
\sqrt{T} I_{T}^{-1 / 2} m_{1}^{S}\left(\hat{\rho}_{T}^{S}, \hat{\theta}_{1 T}(\pi)\right) \Rightarrow & -\left[\frac{B(\pi)}{\pi}-\frac{1}{\sqrt{S}}\left(\frac{B(\pi)^{S}}{\pi}\right)+\frac{I^{-1 / 2} J H(\pi)}{\pi}\right] \\
& +I^{-1 / 2} M_{\rho}\left(M_{\rho}^{\prime} I^{-1} M_{\rho}\right)^{-1} M_{\rho} I^{-1 / 2}\left[B(1)-\frac{1}{\sqrt{S}} B(1)^{S}+I^{-1 / 2} J H(1)\right] .
\end{aligned}
$$

The asymptotic distribution for the second sample can be obtained in a similar way.

\section{Proof of Theorem 4.1:}

First, we show the asymptotic distribution of the estimator $\hat{\theta}_{N}$ obtained with the simulated series for $\rho$ fixed to the restricted estimator $\hat{\rho}_{T}^{S}$. The mean value expansion of the score of the auxiliary model evaluated at $\hat{\theta}_{N}\left(\hat{\rho}_{T}^{S}\right)$ is given by: 


$$
\begin{aligned}
I_{T}^{-1 / 2} \frac{1}{N} \sum_{t=1}^{N} s_{t}\left(\hat{\rho}_{N}^{S}, \hat{\theta}_{T}\right)= & I_{T}^{-1 / 2} \frac{1}{N} \sum_{t=1}^{N} s_{t}\left(\theta^{*}, \rho^{0}\right)+I_{T}^{-1 / 2} \frac{1}{N} \sum_{t=1}^{N} \frac{\partial s_{t}}{\partial \theta^{\prime}}\left(\tilde{\theta}, \hat{\rho}_{T}^{S}\right)\left(\hat{\theta}_{N}\left(\hat{\rho}_{T}^{S}\right)-\theta^{*}\right) \\
& +I_{T}^{-1 / 2} \frac{1}{N} \sum_{t=1}^{N} \frac{\partial s_{t}}{\partial \rho^{\prime}}\left(\hat{\theta}_{N}, \tilde{\rho}\right)\left(\hat{\rho}_{T}^{S}-\rho^{0}\right)+o_{p}(1) .
\end{aligned}
$$

By the asymptotic distribution of the restricted estimator $\hat{\rho}_{T}^{S}$ given in E.3, Assumption A.2, D.1 and the consistency of $\tilde{\theta}$ and $\tilde{\rho}$, we obtain that

$$
\begin{aligned}
\sqrt{N}\left(\hat{\theta}_{N}\left(\hat{\rho}_{T}^{S}\right)-\theta^{*}\right) \Rightarrow & J^{-1} I^{1 / 2} B(1)+\frac{\sqrt{N}}{\sqrt{T}} J^{-1} M_{\rho}\left(M_{\rho}^{\prime} I^{-1} M_{\rho}\right)^{-1} M_{\rho} I^{-1 / 2} \\
& {\left[B(1)-\frac{1}{\sqrt{S}} B(1)^{S}+I^{-1 / 2} J H(1)\right] . }
\end{aligned}
$$

The mean value expansion of the score evaluated at $\hat{\theta}_{N}$ for the data under the alternative is

$$
I_{T}^{-1 / 2} m_{1}\left(\hat{\theta}_{N}\left(\hat{\rho}_{T}^{S}\right), \pi\right)=I_{T}^{-1 / 2} m_{1}^{S}\left(\theta^{*}, \pi\right)+I_{T}^{-1 / 2} \frac{\partial m_{1}}{\partial \theta^{\prime}}(\tilde{\theta}, \pi)\left(\hat{\theta}_{N}\left(\hat{\rho}_{T}^{S}\right)-\theta^{*}\right)+o_{p}(1) .
$$

By the asymptotic distribution of the $\hat{\theta}_{N}$ derived above, Assumptions A.2 and D.1 and the consistency of $\tilde{\theta}$, we obtain under the alternative that

$$
\begin{aligned}
\pi \sqrt{T} I_{T}^{-1 / 2} m_{1}\left(\hat{\theta}_{T}, \pi\right)= & B(\pi)+I^{-1 / 2} J H(\pi)-\pi \frac{\sqrt{T}}{\sqrt{N}} B(1) \\
& -\pi I^{-1 / 2} M_{\rho}\left(M_{\rho}^{\prime} I^{-1} M_{\rho}\right)^{-1} M_{\rho} I^{-1 / 2} \\
& {\left[B(1)-\frac{1}{\sqrt{S}} B(1)^{S}+I^{-1 / 2} J H(1)\right] . }
\end{aligned}
$$




\section{References}

[1] Andrews, D.W.K. (1992), "Generic Uniform Convergence," Econometric Theory, 8, 241-257.

[2] Andrews, D.W.K. (1993), "Tests for Parameter Instability and Structural Change with Unknown Change Point," Econometrica, 61, 821-856.

[3] Andrews, D.W.K. (1994), "The Large Sample Correspondence between Classical Hypothesis Tests and Bayesian Posterior Odds Tests," Econometrica, 62, 1207-1232.

[4] Andrews, D.W.K. and R. Fair (1988), "Inference in Econometrics Models with Structural Change," Review of Econmic Studies, 55, 615-640.

[5] Andrews, D.W.K. and J.C. Monahan (1992), "An Improved Heteroskedasticity and Autocorrelation Consistent Covariance Matrix Estimator," Econometrica, 60, 953-966.

[6] Andrews, D.W.K. and W. Ploberger (1994), "Optimal Tests when a Nuisance Parameter is Present Only under the Alternative," Econometrica, 62, 1383-1414.

[7] Bierens, H. and W. Ploberger (1997) "Asymptotic Theory of Integrated Conditional Moment Tests" Econometrica, 65, 1129-1152.

[8] Bilio, M and A. Monfort (1996), "Functional Indirect Inference", manuscript, CREST, Paris.

[9] Broze, L., R. Dridi and E. Renault (1998), "Calibration of Structural Models by Indirect Inference," Discussion Paper.

[10] Chow, G. C. (1960), "Tests of Equality between Sets of Coefficients in Two Linear Regressions," Econometrica, 28, 591-605.

[11] Davidson, J. (1994), "Stochastic Limit Theory: An Introduction for Econometricians," Oxford University Press, Oxford.

[12] de Jong, R. M. (1996) "The Bierens test under data dependence", Journal of Econometrics 72, 1-32.

[13] Duffie, D. and K. J. Singleton (1993), "Simulated Moments Estimation of Markov Models of Asset Prices," Econometrica, 61, 929-952. 
[14] Dufour, J.M., E. Ghysels and A. Hall (1994), "Generalized Predictive Tests and Structural Change Analysis in Econometrics," International Economic Review, 35, 199-229.

[15] Fenton, V.M. and A.R. Gallant (1996), "Qualitative and Asymptotic Performance of SNP Density Estimators," Journal of Econometrics, 74, 77-118.

[16] Gallant, A.R. (1987), "Nonlinear Statistical Models," John Wiley, New York.

[17] Gallant, A.R. and J.R. Long (1997), "Estimating Stochastic Differential Equations Efficiently by Minimum Chi-Square," Biometrika, 84, 125-141.

[18] Gallant, A.R. and G. Tauchen (1989), "Seminonparametric Estimation of Conditionally Constrained Heterogeneous Processes: Asset Pricing Applications," Econometrica, 57, 1091-1120.

[19] Gallant, A.R. and G. Tauchen (1996), "Which Moments to Match?" EconometricTheory, 12, 657-681.

[20] Gallant, A.R. and G. Tauchen (1997), "EMM: A Program for Efficient Method of Moments Estimation, Version 1.4, User's Guide," Discussion Paper, University of North Carolina at Chapel Hill.

[21] Gallant, A.R. and G. Tauchen (1998), "Reprojecting Partially Observed Systems with Application to Interest Rate Diffusions," Journal of American Statistical Association,93, 10-24.

[22] Gallant, A.R. and H. White (1988), "A Unified Theory of Estimation and Inference for Nonlinear Dynamic Models," Basil Blackwell, New York.

[23] Ghysels, E., A. Guay and A. Hall (1997), "Predictive Test for Structural Change with Unknown Breakpoint," Journal of Econometrics, 82, 209-233.

[24] Ghysels, E., A. Guay (2001), "Structural change tests for Simulated Method of Moments," Discussion Paper.

[25] Ghysels, E. and A. Hall (1990a), "A Test for Structural Stability of Euler Conditions Parameters Estimated Via the Generalized Method of Moments Estimator," International Economic Review, 31, 355-364.

[26] Gouriéroux, C. and A. Monfort (1996), "Simulation-based Econometric Methods," Oxford University Press, Oxford. 
[27] Gouriéroux, C., A. Monfort and E. Renault (1993), "Indirect Inference" Journal of Applied Econometrics, 8, S85-S118.

[28] Gouriéroux, C., A. Monfort and A. Trognon (1985), "Moindres Carrés Asymptotiques," Annales de l'INSEE, 58, 91-121.

[29] Guay, A. (1996), "Structural Change Tests based on Moment Conditions," Discussion Paper UQAM.

[30] Guay, A. and E. Renault (2001), "Indirect Encompassing under misspecification," Discussion Paper.

[31] Hall, A. and A. Sen (1999), "Structural Stability Testing in Models Estimated by Generalized Method of Moments," Journal of Business and Economic Statistics 17, 335-348.

[32] Hansen, L.P. (1982), "Large Sample Properties of Generalized Method of Moments Estimators," Econometrica, 50, 1029-1054.

[33] Hoffman, D. and A. Pagan (1989), "Post-Sample Prediction Tests for Generalized Method of Moments Estimators," Oxford Bulletin of Economics and Statistics, 51, 331-343.

[34] Kodde, D.A., F.C. Palm and G.A. Pfann (1990), "Asymptotic Least-Squares Estimation Efficiency Considerations and Applications," Journal of Applied Econometrics, 5, 229243.

[35] Liu, M. and H.H. Zhang (1998), "Specification Tests in the Efficient Method of Moments Framework with Application to the Stochastic Volatility Models," Discussion Paper, Carnegie Mellon University.

[36] McFadden (1989), "A Method of Simulated Moments for Estimation of Discrete Response Models without Numerical Integration," Econometrica, 57, 995-1026.

[37] Newey, W.K. (1991), "Uniform Convergence in Probability and Stochastic Equicontinuity," Econometrica, 59, 703-708.

[38] Newey, W.K. and K. West (1994), "Automatic Lag Selection in Covariance Matrix Estimation," Review of Economic Studies, 61, 631-653.

[39] Pakes, A. and D. Pollard (1984), "Simulation and the Asymptotic of Optimization Estimators," Econometrica, 57, 1027-1058. 
[40] Pollard, D. (1984), "Convergence of Stochastic Processes," Springer-Verlag, New York.

[41] Potscher, B.M. and Prucha, I. R. (1991), "Basic Structure of the Asymptotic Theory in Dynamic Nonlinear Econometric Model, Part I: Consistency and Approximation Concepts," Econometric Reviews, 10, 125-216.

[42] Sowell, F. (1996a), "Optimal Tests for Parameter Instability in the Generalized Method of Moments Framework," Econometrica, 64, 1085-1107.

[43] Sowell, F. (1996b), "Tests for Violations of Moment Conditions," Manuscript, Graduate School of Industrial Administration, Carnegie Mellon University.

[44] van der Sluis, P. J. (1998), "Structural Stability Tests with Unknown Breakpoint for the Efficient Method of Moments with Application to Stochastic Volatility Models," Manuscript, University of Amsterdam, department of Econometrics. 


\section{Liste des publications au CIRANO*}

Série Scientifique / Scientific Series (ISSN 1198-8177)

2001s-52 The Aftermarket Performance of Initial Public Offerings in Canada / Maher Kooli et Jean-Marc Suret

2001s-51 Capital Structure and Risk Management / Karine Gobert

2001s-50 The Underpricing of Initial Public Offerings: Futher Canadian Evidence / Maher Kooli et Jean-Marc Suret

2001s-49 How Innovative Are Canadian Firms Compared to Some European Firms? A Comparative Look at Innovation Surveys / Pierre Mohnen et Pierre Therrien

2001s-48 A Tale of Two Ports / Ngo Van Long et Kar-yiu Wong

2001s-47 Wage Policy of Firms: An Empirical Investigation / Stéphanie Lluis

2001s-46 Forecasting Some Low-Predictability Time Series Using Diffusion Indices / Marc Brisson, Bryan Campbell et John W. Galbraith

2001s-45 The Importance of the Loss Function in Option Pricing / Peter Christoffersen et Kris Jacobs

2001s-44 Let's Get "Real" about Using Economic Data / Peter Christoffersen, Eric Ghysels et Norman R. Swanson

2001s-43 Fragmentation, Outsourcing and the Service Sector / Ngo Van Long, Ray Riezman et Antoine Soubeyran

2001s-42 Nonlinear Features of Realized FX Volatility / John M. Maheu et Thomas H. McCurdy

2001s-41 Job Satisfaction and Quits: Theory and Evidence from the German Socioeconomic Panel / Louis Lévy-Garboua, Claude Montmarquette et Véronique Simonnet

2001s-40 Logique et tests d'hypothèse : réflexions sur les problèmes mal posés en économétrie / Jean-Marie Dufour

2001s-39 Managing IT Outsourcing Risk: Lessons Learned / Benoit A. Aubert, Suzanne Rivard et Michel Patry

2001s-38 Organizational Design of R\&D Activities / Stefan Ambec et Michel Poitevin

2001s-37 Environmental Policy, Public Interest and Political Market / Georges A. Tanguay, Paul Lanoie et Jérôme Moreau

2001s-36 Wealth Distribution, Entrepreneurship and Intertemporal Trade / Sanjay Banerji et Ngo Van Long

2001s-35 Comparaison des politiques de rémunération en fonction des stratégies organisationnelles / Michel Tremblay et Denis Chênevert

2001s-34 Déterminants et efficacité des stratégies de rémunération : Une étude internationale des entreprises à forte intensité technologique / Michel Tremblay, Denis Chênevert et Bruno Sire

* Consultez la liste complète des publications du CIRANO et les publications elles-mêmes sur notre site Internet : 